TPI-MINN-98/20-T

UMN-TH-1724/98

hep-th/9810068

\title{
Anomaly and Quantum Corrections to Solitons in Two-Dimensional Theories with Minimal Supersymmetry
}

\author{
M. Shifman, A. Vainshtein \\ Theoretical Physics Institute, University of Minnesota, Minneapolis, MN 55455 \\ and \\ M. Voloshin \\ Theoretical Physics Institute, University of Minnesota, Minneapolis, MN 55455 \\ and \\ Institute of Experimental and Theoretical Physics, Moscow 117259
}

\begin{abstract}
We reexamine the issue of the soliton mass in two-dimensional models with $\mathcal{N}=1$ supersymmetry. The superalgebra has a central extension, and at the classical level the soliton solution preserves $1 / 2$ of supersymmetry which is equivalent to BPS saturation. We prove that the property of BPS saturation, i.e. the equality of the soliton mass to the central charge, remains intact at the quantum level in all orders of the weak coupling expansion. Our key finding is an anomaly in the expression for the central charge. The classical central charge, equal to the jump of the superpotential, is amended by an anomalous term proportional to the second derivative of the superpotential. The anomaly is established by various methods in explicit one-loop calculations. We argue that this one-loop result is not affected by higher orders. We discuss in detail how the impact of the boundary conditions can be untangled from the soliton mass calculation. In particular, the soliton profile and the energy distribution are found at one loop. A "supersymmetry" in the soliton mass calculations in the non-supersymmetric models is observed.
\end{abstract}

Phys. Rev. D, to appear 


\section{Contents}

$\begin{array}{lll}1 & \text { Introduction } & 2\end{array}$

\begin{tabular}{|lll}
2 & The minimal SUSY in two dimensions & 6
\end{tabular}

2.1 Superspace for $\mathcal{N}=1$ SUSY models . . . . . . . . . . . . . . . . . . . . 6

2.2 Multiple vacua and kinks . . . . . . . . . . . . . . . . . . . . . 7

2.3 Witten's index in the models with minimal supersymmetry . . . . . . . 10

$\begin{array}{lll}3 & \text { Ultraviolet and infrared regularization } & 11\end{array}$

3.1 Superalgebra (unregularized) . . . . . . . . . . . . . . . . . . . . . . 11

3.2 Anomaly in the central charge . . . . . . . . . . . . . . . . . . . . . 12

3.3 Representations of the centrally extended algebra . . . . . . . . . . . . 15

3.4 Ultraviolet regularization by higher derivatives . . . . . . . . . . . . . . 16

3.5 Calculation of the anomaly by higher-derivative regularization . . . . . 17

3.6 One-loop nature of the anomaly . . . . . . . . . . . . . . . . . . . 18

3.7 Infrared regularization and fermion-boson cancellations in local quantities 19

3.8 Explicit expressions for the modes and Green's functions . . . . . . . . 22

3.9 Separating soliton from the boundaries . . . . . . . . . . . . . . . . . . 24

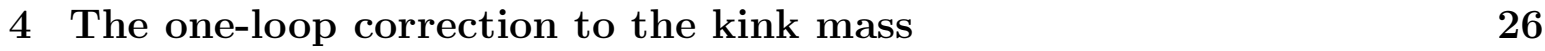

4.1 Central charge and soliton mass at one loop . . . . . . . . . . . . . . . . 26

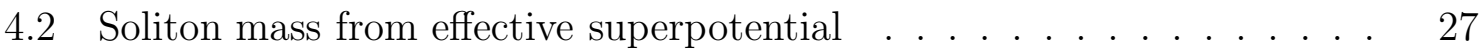

5 Field and energy profile at one loop $\quad 30$

5.1 General relations for profiles . . . . . . . . . . . . . . . . . . . 30

5.2 Polynomial model . . . . . . . . . . . . . . . . . . . . . . . . . . . . . . . . . . . . . . . . . .

5.3 Sine-Gordon model . . . . . . . . . . . . . . . . . . . . . . 35

6 "Supersymmetry" of the non-SUSY sine-Gordon model. 36

7 Anomaly and soliton mass in the SSG model at two loops 39

$8 \quad$ Extra superfields and extended supersymmetry $\quad 40$

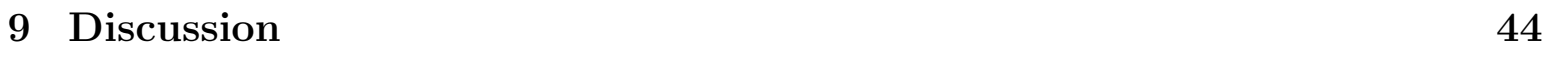

$\begin{array}{ll}\text { Acknowledgments } & 47\end{array}$

$\begin{array}{ll}\text { References } & 48\end{array}$ 


\section{Introduction}

The issue of quantum corrections to the soliton mass in two-dimensional models with $\mathcal{N}=1$ supersymmetry has a long and dramatic history. As was noted in Ref. [1], in the models with central extensions topologically stable solitons can be Bogomol'nyi-PrasdSommerfeld (BPS) saturated. Such solitons are annihilated by the action of $1 / 2$ of the supergenerators, so that $1 / 2$ of the supersymmetry (SUSY) is preserved. The remaining $1 / 2$ of the supergenerators act non-trivially. The mass of the BPS saturated solitons must be equal to the central charge. The simplest two-dimensional model with the minimal superalgebra, admitting solitons, was considered in [2]. This is the Wess-Zumino model with one real scalar field and one two-component real spinor (to be referred to as the minimal model hereafter). It was argued [2] that, due to a residual supersymmetry, the mass of the soliton calculated at the classical level remains intact at the one-loop level. A few years later it was noted [3] that the non-renormalization theorem [2] cannot possibly be correct, since the classical soliton mass is proportional to $m^{3} / \lambda^{2}$ (where $m$ and $\lambda$ are the bare mass parameter and coupling constant, respectively), and the physical mass of the scalar field gets a logarithmically infinite renormalization. Since the soliton mass is an observable physical parameter, it must stay finite in the limit $M_{\mathrm{uv}} \rightarrow \infty$, where $M_{\mathrm{uv}}$ is the ultraviolet cut off. This implies, in turn, that the quantum corrections cannot vanish - they "dress" $m$ in the classical expression, converting the bare mass parameter into the renormalized one. The one-loop renormalization of the soliton mass was first calculated in [3]. Technically the emergence of the one-loop correction was attributed to a "difference in the density of states in continuum in the boson and fermion operators in the soliton background field". The subsequent work [4 dealt with the renormalization of the central charge, with the conclusion that the central charge is renormalized in just the same way as the soliton mass, so that the saturation condition is not violated.

Since then a number of one-loop calculations have been carried out [5]. The results reported and the conclusion of saturation/non-saturation oscillate with time, with no sign of convergence. Although all authors clearly agree that the logarithmically divergent term corresponds to the renormalization of $m$, the finite term comes out differently, varying from work to work, sometimes even in the successive works of the same authors, e.g. [13, 14] or [8, 9]. According to a recent publication [14], the BPS saturation is violated at one loop. This assertion reverses the earlier trend [3, 10, 11], according to which the soliton in the minimal model is BPS saturated: the central charge and the soliton mass are renormalized in a concerted way.

The problem becomes obscure partly due to the fact that some authors (e.g. [3]) use non-supersymmetric boundary conditions. When non-supersymmetric boundary conditions are imposed, explicit supersymmetry is lost. Gone with it is the cancellation of the boson and fermion loops inherent to supersymmetry. Then one has to consider separately the boson and fermion contributions - each of them individually diverges. Extracting finite terms in the difference of two diverging contributions complicates the analysis. This approach - the separate treatment of the boson and fermion corrections 
in a SUSY-insensitive manner - prevails in the recent works. Although such approach is fully legitimate, it does not take advantage of SUSY.

We reexamine the issue of the quantum corrections to solitons in two-dimensional models with minimal supersymmetry. Our strategy is to take maximal advantage of supersymmetry, at every step. One of our main goals is establishing a relation with other similar problems where 1/2 of SUSY is preserved in the background field: the instanton calculations in SUSY gluodynamics [16] and the BPS-saturated domain walls [17, 18]. In both cases the quantum corrections totally cancel, bosons against fermions. Up to a point, a similar cancellation takes place in the two-dimensional models too. If it were not for boundary effects, the classical expression for the soliton mass would not be corrected at the quantum level. The boundary effects, which play no role in SUSY instantons or the BPS-saturated domain walls, are responsible for the soliton mass renormalization in the two-dimensional models with minimal supersymmetry. The divergence between the two- and four-dimensional problems lies here. A non-renormalization theorem survives in the form of the statement that the soliton mass is equal to the central charge, to all orders. We present a general argument for why the BPS saturation cannot be violated by quantum corrections in the weak coupling regime. In four dimensions the phenomenon known as multiplet shortening protects the BPS states from becoming nonsaturated at higher orders. In two dimensions the multiplet shortening does not occur, literally speaking. However, the multiplet shortening per se is not the reason lying behind the protected nature of the BPS states, but, rather, a signature. The genuine reason is the existence of an invariant subalgebra of the superalgebra, i.e. the existence of a supercharge $Q$ possessing the following properties: (i) $Q^{2}$ is nontrivial; (ii) $Q$ annihilates all states from the given multiplet. Although the multiplet shortening does entail the existence of such a subalgebra, the inverse is not necessarily true. In fact, the two-dimensional models with minimal supersymmetry present an example of the problem where the invariant subalgebra of the required type exists without the multiplet shortening. This is sufficient for establishing the theorem "soliton mass $=$ central charge", order by order.

The next question to address is: "what is the central charge in the theories with minimal supersymmetry?" This is a key element of our analysis. As we will show below, much of the confusion in the current literature is due to a tacit assumption that the central charge $\mathcal{Z}$ is equal to the difference between the values of the superpotential $\mathcal{W}$ at spatial infinities,

$$
\mathcal{Z}=\mathcal{W}[\phi(z=\infty)]-\mathcal{W}[\phi(z=-\infty)]
$$

This expression was obtained long ago [1]. At the classical level it is a valid expression. It was implied in the previous studies that the only change at the quantum level is the substitution of the classical superpotential $\mathcal{W}$ in Eq. (1.1 ) by its operator form. We show that the central charge is additionally modified by a quantum anomaly,

$$
\mathcal{W} \longrightarrow \mathcal{W}+\frac{\mathcal{W}^{\prime \prime}}{4 \pi}
$$

i.e. the anomalous term $\mathcal{W}^{\prime \prime}$ should be added in the expression for the central charge. 
We have obtained this anomaly by four different methods: (i) using dimensional regularization; (ii) regularization by higher derivatives; (iii) from an effective superpotential; (iv) from the models with the extended $(\mathcal{N}=2)$ supersymmetry deformed by soft terms breaking of $\mathcal{N}=2$ down to $\mathcal{N}=1$. If the first two methods are quite straightforward, the third and the fourth require comment. As in many other similar problems, it is convenient to calculate the soliton mass starting from an effective Lagrangian (superpotential), including the loop effects by construction, rather than from the bare Lagrangian. It is not difficult to obtain the effective superpotential at one-loop level, which results, in turn, in a certain prediction for the soliton mass. This prediction can be compared with the result obtained through the central charge. The two calculations agree with each other provided one accounts for the anomaly in Eq. (1.2).

An alternative approach is based on the models with extended supersymmetry, $\mathcal{N}=2$. The $\mathcal{N}=2$ theories in two dimensions can be obtained by dimensional reduction of the Wess-Zumino model [19], from four to two dimensions. The superpotential is not renormalized [20], and so neither is the soliton mass. The boundary effects - the prime culprits for the soliton mass renormalization in the minimal model - vanish in the $\mathcal{N}=2$ case. We then deform the $\mathcal{N}=2$ model by introducing terms which softly break $\mathcal{N}=2$ SUSY down to $\mathcal{N}=1$. The emerging dynamics is quite rich and interesting. On the technical side, the softly broken $\mathcal{N}=2$ theories provide a possible regularization procedure for $\mathcal{N}=1$ theories. Applying this procedure we recover Eq. (1.2) again, for the fourth time.

Although the fact that the central charge does have anomalies is very well known in the four-dimensional theories (e.g. [18]), the existence of the anomaly in two-dimensional theories with minimal supersymmetry was overlooked in all previous publications. Needless to say that the additional term proportional to $\mathcal{W}^{\prime \prime}$ in Eq. (1.2) gives a non-vanishing contribution to the central charge. In conjunction with the statement of BPS saturation, this contribution translates into a (finite) extra term in the soliton mass. If the BPS saturation per se is established on the basis of comparison between two distinct calculations - of the soliton mass, on the one hand, and of the central charge on the other - the omission of the anomalous term leads to an apparent paradox. The general theorem forbidding the loss of BPS saturation in quantum loops in the weak coupling expansion seems to be violated.

Since the quantum corrections are associated with boundary effects, their determination reduces to a calculation in the "empty" vacuum, which can be trivially performed. At the same time, the standard calculation of the soliton mass becomes sensitive to the choice of boundary conditions. This is another subtle point which was not properly treated in the literature. It was believed that the soliton mass depends on the boundary conditions, and that particular choices of the boundary conditions are "more correct" than others. It is clear that physically the soliton mass is saturated in the domain near its center, and must not depend on the boundary conditions set at a large distance from the soliton center.

Although the boundary conditions do not affect the physical soliton mass, some choices are practically more convenient. In fact, inappropriate choices contaminate the 
field near the boundary and may shift the total energy in the box by a quantity which is of the same order as the "genuine" one-loop quantum correction to the kink mass. If the boundary domain is not treated properly, the resulting calculation yields the mass of a contaminated soliton, rather than the physical mass of the ground state in the sector with unit topological charge (i.e. the true soliton). Much of the controversy existing in the literature is due to this contamination. We explicitly demonstrate how these distortions appear in the boundary domain, and show how the boundary conditions can be untangled from the soliton mass calculations. One of the possibilities to control the contamination is to consider the energy-density distribution in the soliton at one loop. This is done in Sec. 5. In addition, we perform a calculation of the one-loop correction to the soliton profile.

The last issue addressed in this work is the soliton mass calculation in the nonsupersymmetric sine-Gordon model. Surprisingly, this problem is naturally formulated in supersymmetric terms too, although the original Lagrangian has no explicit supersymmetry. The role of the SUSY partners is played in the case at hand by the sectors with the zero and unit topological charges, respectively. This approach yields a number of useful results. In particular, closed analytical expressions for the boson and fermion Green functions in the soliton background, known previously [21, 22], are given a transparent interpretation.

The outline of the paper is as follows. In Sec. 2 we briefly review the formulation of the problem, and general aspects of two-dimensional theories with minimal supersymmetry. Most of the results presented here are not new. Since they are scattered in the literature we find it convenient to collect them in one place, organizing a common perspective. We briefly review the superfield formalism in two dimensions and describe the two models with minimal supersymmetry we will deal with: the polynomial model and the sine-Gordon model. Then the kink solutions are discussed, and the notion of the kink superfield is introduced. A residual supersymmetry which annihilates the kink is identified. At the classical level, the corresponding SUSY generator acts on the kink trivially. It is shown that this property cannot be lost in perturbation theory. In Sec. 2.3 we discuss Witten's index in two-dimensional models with minimal supersymmetry.

Section 3 is devoted to derivations of the central charge. The focus is on the anomalous part of the central charge, which is obtained in a variety of ways. We formulate a convenient (and universal) regularization procedure based on higher derivatives, which regularizes all ultraviolet divergences. We also explain how the same anomaly emerges as an infrared effect, through the level flow. This is a standard situation - the anomaly has two faces: ultraviolet and infrared (see e.g. [23]). In Sec. 3.7 a local version of the BPS saturation condition is explored. In Sec. 1 the one-loop corrections to the kink mass are calculated, both in general form and in the two models under consideration, the polynomial model and sine-Gordon. We present two distinct calculations. Section 5 deals with the soliton profile at one loop. The energy-density distribution in the kink is also presented here. The results are based on the explicit expressions for Green's function in the soliton background. In Sec. 6 the calculations of the soliton masses in non-supersymmetric polynomial and sine-Gordon models are reformulated in terms 
of a supersymmetry which - surprisingly - can be introduced in these problems. Section 7 presents the calculation of the soliton mass in the supersymmetric sine-Gordon model at two loops. Finally, Sec. 8 is devoted to extended supersymmetry and $\mathcal{N}=1$ deformations of the $\mathcal{N}=2$ model. Section 9 summarizes our findings.

\section{The minimal SUSY in two dimensions}

\subsection{Superspace for $\mathcal{N}=1$ SUSY models}

The two-dimensional space $x^{\mu}=\{t, z\}$ can be promoted to superspace by adding a two-component real Grassmann variable $\theta_{\alpha}=\left\{\theta_{1}, \theta_{2}\right\}$. The coordinate transformations

$$
\theta_{\alpha} \rightarrow \theta_{\alpha}+\varepsilon_{\alpha}, \quad x^{\mu} \rightarrow x^{\mu}-i \bar{\theta} \gamma^{\mu} \varepsilon
$$

add SUSY to the translational and Lorentz invariances. A convenient representation for the two-dimensional Majorana $\gamma$ matrices we use throughout this paper is

$$
\gamma^{0}=\sigma_{2}, \quad \gamma^{1}=i \sigma_{3}
$$

The real superfield $\Phi(x, \theta)$ has the form

$$
\Phi(x, \theta)=\phi(x)+\bar{\theta} \psi(x)+\frac{1}{2} \bar{\theta} \theta F(x),
$$

where $\bar{\theta}=\theta \gamma^{0}$, and $\theta, \psi$ are real two-component spinors. The superspace transformations (2.1) generate the following SUSY transformations:

$$
\delta \phi=\bar{\varepsilon} \psi, \quad \delta \psi=-i \partial_{\mu} \phi \gamma^{\mu} \varepsilon+F \varepsilon, \quad \delta F=-i \bar{\varepsilon} \gamma^{\mu} \partial_{\mu} \psi
$$

In $1+1$ dimensions it is a minimal, $\mathcal{N}=1$, supersymmetry.

The action of a model invariant under the transformations (2.4) is

$$
S=i \int \mathrm{d}^{2} \theta \mathrm{d}^{2} x\left\{\frac{1}{4} \bar{D}_{\alpha} \Phi D_{\alpha} \Phi+\mathcal{W}(\Phi)\right\}
$$

where $\mathcal{W}(\Phi)$ will be referred to as the superpotential, keeping in mind a parallel with the four-dimensional Wess-Zumino model, although in the case at hand the superpotential term is the integral over the full superspace, and is not chiral. Moreover, $\mathrm{d}^{2} x=\mathrm{d} t \mathrm{~d} z$; the spinorial derivatives are defined as follows

$$
D_{\alpha}=\frac{\partial}{\partial \bar{\theta}_{\alpha}}-i\left(\gamma^{\mu} \theta\right)_{\alpha} \partial_{\mu}, \quad \bar{D}_{\alpha}=-\frac{\partial}{\partial \theta_{\alpha}}+i\left(\bar{\theta} \gamma^{\mu}\right)_{\alpha} \partial_{\mu}
$$

so that

$$
\left\{D_{\alpha} \bar{D}_{\beta}\right\}=2 i\left(\gamma^{\mu}\right)_{\alpha \beta} \partial_{\mu}
$$


In components the Lagrangian takes the form

$$
\mathcal{L}=\frac{1}{2}\left(\partial_{\mu} \phi \partial^{\mu} \phi+\bar{\psi} i \not \partial \psi+F^{2}\right)+\mathcal{W}^{\prime}(\phi) F-\frac{1}{2} \mathcal{W}^{\prime \prime}(\phi) \bar{\psi} \psi
$$

In two dimensions any superpotential function $\mathcal{W}$ leads to a renormalizable field theory and is thus allowed. Many of the results presented below are independent of the particular choice of $\mathcal{W}$. For our purposes it is generally sufficient to limit ourselves to odd functions of $\Phi$, i.e. $\mathcal{W}(\Phi)=-\mathcal{W}(-\Phi)$ (Sec. 8 is exception). We require the superpotential $\mathcal{W}(\phi)$ to have more than one extremum, i.e. the equation $\mathrm{d} \mathcal{W} / \mathrm{d} \phi=$ 0 must have at least two solutions. Then one deals with several degenerate vacua. The field configurations interpolating between distinct vacua at the spatial infinities $(z \rightarrow \pm \infty)$ are topologically stable solitons (kinks).

In certain instances we find it illustrative to consider specific examples. Two choices are of most practical interest: the polynomial superpotential, and the minimal supergeneralization of the sine-Gordon model. In the first case the superpotential function $\mathcal{W}$ is parameterized as follows:

$$
\mathcal{W}(\Phi)=\frac{m^{2}}{4 \lambda} \Phi-\frac{\lambda}{3} \Phi^{3}
$$

where the parameters $m$ and $\lambda$ are real numbers. In the second case

$$
\mathcal{W}(\Phi)=m v^{2} \sin \frac{\Phi}{v}
$$

The first model will be referred to as SPM, the second as SSG.

\subsection{Multiple vacua and kinks}

The SPM model has two vacuum states corresponding to $\phi_{*}^{ \pm}= \pm m /(2 \lambda)$. A kink solution interpolates between $\phi_{*}^{-}=-m / 2 \lambda$ at $z \rightarrow-\infty$ and $\phi_{*}^{+}=m / 2 \lambda$ at $z \rightarrow \infty$, while an antikink between $\phi_{*}^{+}=m / 2 \lambda$ and $\phi_{*}^{-}=-m / 2 \lambda$. The classical kink solution has the form

$$
\phi_{0}=\frac{m}{2 \lambda} \tanh \frac{m z}{2} .
$$

In the sine-Gordon model there are infinitely many vacua; they lie at

$$
\phi_{*}^{k}=v\left(\frac{\pi}{2}+k \pi\right)
$$

where $k$ is an integer, either positive or negative. Correspondingly, there exist solitons connecting any pair of vacua. In this case we will limit ourselves to consideration of the "elementary" solitons connecting the adjacent vacua, e.g. $\phi_{*}^{0,-1}= \pm \pi v / 2$,

$$
\phi_{0}=v \arcsin [\tanh (m z)]
$$


Both solutions (2.10) and (2.12) are BPS saturated, i.e. they satisfy the first order differential equation,

$$
\frac{\mathrm{d} \phi_{0}(z)}{\mathrm{d} z}=-F\left(\phi_{0}\right)=\mathcal{W}^{\prime}\left(\phi_{0}\right)
$$

In terms of superfields the BPS saturation means that a background field $\Phi_{0}$ is of the form

$$
\Phi_{0}(x, \theta)=f\left(z-\frac{1}{2} \bar{\theta} \theta\right),
$$

where $f$ is some function. In other words, instead of the generic superfield $\Phi(x, \theta)$ depending on two coordinates $x^{\mu}$ and two Grassmann coordinates $\theta_{\alpha}$ we deal with a background (super)field depending only on one specific combination, $z-\bar{\theta} \theta / 2$. In components Eq. (2.14) implies that

$$
\phi_{0}=f(z), \quad F_{0}=-\frac{\mathrm{d} f(z)}{\mathrm{d} z} .
$$

The soliton solutions (2.10) and (2.12) belong to this class, with a special choice of the function $f(z)$.

A special, topological nature of the background field (2.14) is seen from the following property: the total energy is defined by the asymptotics at $z \rightarrow \pm \infty$,

$$
-\int \mathcal{L}\left[\Phi_{0}\right] d z=\Delta \mathcal{W} \equiv \mathcal{W}[\phi(z=\infty)]-\mathcal{W}[\phi(z=-\infty)]
$$

irrespective of the particular choice of the function $f$. Substituting the asymptotic values of $\phi$ at $\pm \infty$ in Eq. (2.16) we find the soliton mass

$$
M_{0}= \begin{cases}\frac{m^{3}}{6 \lambda^{2}} & (\mathrm{SPM}) \\ 2 m v^{2} & (\mathrm{SSG})\end{cases}
$$

where the subscript 0 emphasizes that this result is obtained at the classical level.

For any background field of the type (2.14) half of the supersymmetry is preserved. Indeed, let us consider supertransformations (2.4) with the parameter $\varepsilon_{2}=0$,

$$
\delta \theta_{1}=\varepsilon_{1}, \quad \delta \theta_{2}=0, \quad \delta t=-i \theta_{1} \varepsilon_{1}, \quad \delta z=i \theta_{2} \varepsilon_{1} .
$$

This transformation leaves the interval

$$
z_{\mathrm{inv}} \equiv z-\frac{1}{2} \bar{\theta} \theta=z+i \theta_{1} \theta_{2}
$$

invariant. Consequently, backgrounds of the form (2.14) are not changed. It means that quantization preserves the invariance under the following transformations of the fields,

$$
\delta\left[\begin{array}{c}
\phi \\
-i \psi_{2}
\end{array}\right]=\varepsilon_{1}\left[\begin{array}{c}
-i \psi_{2} \\
-i \dot{\phi}
\end{array}\right], \quad \delta\left[\begin{array}{c}
\psi_{1} \\
\partial_{z} \phi+F
\end{array}\right]=\varepsilon_{1}\left[\begin{array}{c}
\partial_{z} \phi+F \\
-i \dot{\psi}_{1}
\end{array}\right]
$$


This form shows the multiplet structure of the surviving supersymmetry. An important consequence of the invariance (2.20) is that the fermion-boson degeneracy is not lost for quantum excitations although a part of SUSY is broken in the given fixed background.

The part of supersymmetry which is spontaneously broken by the soliton configuration corresponds to the transformations produced by $\varepsilon_{2}$,

$$
\delta \theta_{1}=0, \quad \delta \theta_{2}=\varepsilon_{2}, \quad \delta t=-i \theta_{2} \varepsilon_{2}, \quad \delta z=i \theta_{1} \varepsilon_{2} .
$$

Applied to the soliton solution it generates the fermionic field $\psi_{2}$ (use Eq. (2.4) with $\left.\varepsilon_{1}=0\right)$ :

$$
\delta \psi_{2}=\left(-\partial_{z} \phi_{0}+F_{0}\right) \varepsilon_{2}=-2 \frac{\mathrm{d} \phi_{0}(z)}{\mathrm{d} z} \varepsilon_{2} .
$$

In addition to the above fermion zero mode there is also the bosonic zero mode generated by the spatial translation. Both zero modes can be conveniently described by the introduction of two collective coordinates: the soliton center $z_{0}$ and the real Grassmann parameter $\eta$ corresponding to the fermion zero mode, in much the same way this was done for instantons [24] and domain walls [18]. To this end let us make the substitution

$$
z_{\mathrm{inv}}=z+i \theta_{1} \theta_{2} \quad \longrightarrow \quad \tilde{z}_{\mathrm{inv}}=z-z_{0}+i \theta_{1}\left(\theta_{2}-\eta\right) \text {. }
$$

The kink superfield, with all collective coordinates included, is

$$
\Phi_{0}=f\left(\tilde{z}_{\text {inv }}\right)
$$

where the function $f$ is given by Eq. (2.10) for the polynomial model and by Eq. (2.12) for the sine-Gordon one.

The deformation of the kink superfield $\Phi_{0}$ under the supertransformations (2.1) is compensated by the following transformations of the collective coordinates:

$$
\delta z_{0}=i \eta \varepsilon_{1}, \quad \delta \eta=2 \varepsilon_{2} .
$$

The parameters $z_{0}$ and $\eta$ can be treated as time-dependent dynamical variables, describing the superkink. The quantum mechanics of these coordinates emerges upon integration over all non-zero modes in the field-theoretical model at hand,

$$
\begin{gathered}
Z=\int \mathcal{D}[\phi(x)] \mathcal{D}[\psi(x)] \exp \left\{i \int \mathcal{L}(\phi, \psi) \mathrm{d}^{2} x\right\}= \\
\int \mathcal{D}\left[z_{0}(t)\right] \mathcal{D}[\eta(t)] \exp \left\{i \int \mathrm{d} t\left[-M+\frac{1}{2} M \dot{z}_{0}^{2}+\frac{i M}{2} \eta \dot{\eta}\right]\right\}
\end{gathered}
$$

where the functional integral is performed in the sector with unit kink number, and $M$ is the physical mass of the kink. 


\subsection{Witten's index in the models with minimal supersymme- try}

Here we comment on a general aspect of the two-dimensional models with minimal supersymmetry which is only indirectly related to the solitons. In the model (2.5), (2.7) with the polynomial potential the Witten index is zero. This can be seen in many ways. Say, if the mass parameter $m^{2}$ in the superpotential (2.8) is made negative, the equation $\mathrm{d} \mathcal{W} / \mathrm{d} \Phi=0$ has no real solutions. At negative $m^{2}$ the $Z_{2}$ symmetry

$$
\phi \rightarrow-\phi, \quad \psi \rightarrow \gamma_{5} \psi
$$

stays unbroken. This protects $\phi$ from developing a vacuum expectation value. Therefore, the $\psi$ field is massless, and is the Goldstino of the spontaneously broken SUSY. A convenient order parameter is $\left\langle\mathcal{W}^{\prime}\right\rangle$, where

$$
\mathcal{W}^{\prime} \equiv \frac{\mathrm{d} \mathcal{W}}{\mathrm{d} \Phi}=\frac{m^{2}}{4 \lambda}-\lambda \Phi^{2}
$$

Since $\mathcal{W}^{\prime}=(1 / 2) \bar{D} D \Phi$, the expectation value $\left\langle\mathcal{W}^{\prime}\right\rangle=0$ in the supersymmetric vacuum.

Alternatively, one can consider the theory with positive $m^{2}$ in a finite box. If, following Witten [25], we make the box width $L$ small, $\lambda L \ll 1$, and retain only the zero momentum modes, discarding all others, we get a quantum-mechanical system known to have a vanishing Witten index [25].

At positive (and large) values of $m^{2}$ the $Z_{2}$ symmetry is spontaneously broken, so that the theory has two physically equivalent vacua. The vanishing of the Witten index implies that if one of the vacua is bosonic, the other is fermionic. This is a unique feature of the minimal model: in four-dimensional field theories and in two-dimensional field theories with extended supersymmetry (and no massless particles) all vacuum states are clearly of the bosonic type.

All theories with vanishing Witten index are potentially unstable with respect to spontaneous SUSY breaking. Moreover, in a finite volume this spontaneous breaking does occur. However, in the infinite volume limit spontaneous SUSY breaking is impossible provided $\lambda^{2} / m^{2} \ll 1$. Indeed, one can show that $\lambda^{2} / m^{2}$ is the genuine dimensionless expansion parameter in the theory at hand. At small $\lambda^{2} / m^{2}$ the theory is weakly coupled. Since there are no massless fields in the Lagrangian, (and they cannot appear as bound states in the weak coupling regime), there is no appropriate candidate to play the role of Goldstino, and, hence, supersymmetry must be realized linearly.

The Witten index for the sine-Gordon model also vanishes. This can be checked by putting the theory in a finite box. The resulting quantum mechanics has two supersymmetric vacua - one bosonic and one fermionic - provided the wave functions considered are periodic. One can lift these two vacua from zero by imposing Bloch boundary conditions on the wave functions, with the angle $\theta \neq 0$. 


\section{Ultraviolet and infrared regularization}

\subsection{Superalgebra (unregularized)}

We start with the operator construction which corresponds to the classical level of consideration. The SUSY algebra is built on the supercharges $Q_{\alpha}$, defined as

$$
Q_{\alpha}=\int \mathrm{d} z J_{\alpha}^{0}
$$

where the conserved supercurrent is

$$
J^{\mu}=\left(\partial_{\nu} \phi\right) \gamma^{\nu} \gamma^{\mu} \psi-i F \gamma^{\mu} \psi
$$

Using the canonical commutation relations 1

$$
\left[\phi(t, z), \dot{\phi}\left(t, z^{\prime}\right)\right]=i \delta\left(z-z^{\prime}\right), \quad\left\{\psi_{\alpha}(t, z), \bar{\psi}_{\beta}\left(t, z^{\prime}\right)\right\}=\left(\gamma^{0}\right)_{\alpha \beta} \delta\left(z-z^{\prime}\right)
$$

one finds the anticommutator,

$$
\left\{J_{\alpha}^{\mu}, \bar{Q}_{\beta}\right\}=2\left(\gamma_{\nu}\right)_{\alpha \beta} \vartheta^{\mu \nu}+2 i\left(\gamma^{5}\right)_{\alpha \beta} \zeta^{\mu}
$$

where $\vartheta^{\mu \nu}$ is the energy-momentum tensor

$$
\vartheta^{\mu \nu}=\partial^{\mu} \phi \partial^{\nu} \phi+\frac{1}{2} \bar{\psi} \gamma^{\mu} i \partial^{\nu} \psi-\frac{1}{2} g^{\mu \nu}\left[\partial_{\gamma} \phi \partial^{\gamma} \phi-F^{2}\right],
$$

and $\zeta^{\mu}$ is the conserved topological current,

$$
\zeta^{\mu}=\epsilon^{\mu \nu} \partial_{\nu} \mathcal{W}
$$

Symmetrization (antisymmetrization) over the bosonic (fermionic) operators in the products is implied in the above expressions. Also, the notation, $\gamma^{5}=\gamma^{0} \gamma^{1}=-\sigma_{1}$ and $F=-\mathcal{W}^{\prime}$, is used.

Integrating the $\mu=0$ component of Eq. (3.4) over space gives the SUSY algebra:

$$
\left\{Q_{\alpha}, \bar{Q}_{\beta}\right\}=2\left(\gamma^{\mu}\right)_{\alpha \beta} P_{\mu}+2 i\left(\gamma^{5}\right)_{\alpha \beta} \mathcal{Z}
$$

Here $P_{\mu}=\int \mathrm{d} z \vartheta_{0 \mu}$ are operators of the total energy and momentum, and $\mathcal{Z}$ is the central charge,

$$
\mathcal{Z}=\int \mathrm{d} z \zeta^{0}=\int \mathrm{d} z \partial_{z} \mathcal{W}(\phi)=\mathcal{W}[\phi(z=\infty)]-\mathcal{W}[\phi(z=-\infty)]
$$

which coincides with the topological one. In the theories (2.7) with several degenerate vacua the central charge $\mathcal{Z}$ is nontrivial for the soliton configurations. Thus, the $\mathcal{N}=1$ superalgebra gets centrally extended [1].

Let us emphasize that Eq. (3.4) establishes supersymmetry relations between the supercurrent $J_{\mu}$, the energy-momentum tensor $\vartheta_{\mu \nu}$, and the topological current $\zeta_{\mu}$, and for this reason cannot be changed by quantum corrections. The explicit expressions (3.2), (3.5) and (3.6) for $\vartheta_{\mu \nu}$ and $\zeta_{\mu}$ are classical ones, quantum corrections will change them.

\footnotetext{
${ }^{1}$ Here and below $\dot{\phi}$ should be understood as the canonical momentum $\pi_{\phi}$ conjugate to $\phi$.
} 


\subsection{Anomaly in the central charge}

The structure of the centrally extended superalgebra (3.7) is quite general. At the same time, the particular expression for the central charge depends on the dynamics of the model under consideration. Equation (3.8) is obtained at the classical level. It is easy to see that quantum corrections do modify it. Here we will present a simple argument demonstrating the emergence of an anomalous term in the central charge. We also discuss its physical meaning. A detailed direct calculation will be carried out after we introduce the ultraviolet regularization by higher derivatives.

To begin with, let us consider $\gamma^{\mu} J_{\mu}$ where $J_{\mu}$ is the supercurrent defined in Eq. (3.2). This quantity is related to the superconformal properties of the model under consideration. At the classical level

$$
\left(\gamma^{\mu} J_{\mu}\right)_{\text {class }}=-2 i F \psi \text {. }
$$

Note that the first term in the supercurrent (3.2) gives no contribution in Eq. (3.9) due to the fact that in two dimensions $\gamma_{\mu} \gamma^{\nu} \gamma^{\mu}=0$.

Multiplying Eq. (3.4) by $\gamma_{\mu}$ from the left we get the supertransformation of $\gamma_{\mu} J^{\mu}$,

$$
\frac{1}{2}\left\{\gamma^{\mu} J_{\mu}, \bar{Q}\right\}=\vartheta_{\mu}^{\mu}+i \gamma_{\mu} \gamma^{5} \zeta^{\mu}
$$

This equation establishes the supersymmetric relation between $\gamma^{\mu} J_{\mu}, \vartheta_{\mu}^{\mu}$ and $\zeta^{\mu}$ and, as was mentioned above, remains valid with quantum corrections included. But expressions for these operators could be changed. Classically the trace of the energymomentum tensor is

$$
\left(\vartheta_{\mu}^{\mu}\right)_{\text {class }}=F^{2}+\frac{1}{2} \mathcal{W}^{\prime \prime} \bar{\psi} \psi
$$

as follows from Eq. (3.5). The zero component of $\zeta^{\mu}$ in the second term classically coincides with the density of the central charge, $\partial_{z} \mathcal{W}$, see Eq. (3.6). It is seen that the trace of the energy-momentum tensor and the density of the central charge appear in this relation together.

It is well-known that in renormalizable theories with ultraviolet logarithmic divergences, both the trace of the energy-momentum tensor and $\gamma^{\mu} J_{\mu}$ have anomalies. We will use this fact, in conjunction with Eq. (3.10), to establish the general form of the anomaly in the density of the central charge.

To get some idea of the anomaly, it is convenient to use the dimensional regularization. If we assume that the number of dimensions $D=2-\varepsilon$ rather than $D=2$ the first term in Eq. (3.2) does generate a nonvanishing contribution to $\gamma^{\mu} J_{\mu}$, proportional to $(D-2)\left(\partial_{\nu} \phi\right) \gamma^{\nu} \psi$. At the quantum level this operator gets an ultraviolet logarithm (i.e. $(D-2)^{-1}$ in dimensional regularization), so that $D-2$ cancels, and we are left with an anomalous term in $\gamma^{\mu} J_{\mu}$.

To do the one-loop calculation we apply here (and in many instances below) the background field technique: we substitute the field $\phi$ by its background and quantum parts, $\phi$ and $\chi$, respectively,

$$
\phi \longrightarrow \phi+\chi
$$


Specifically, for the anomalous term in $\gamma^{\mu} J_{\mu}$,

$$
\left(\gamma^{\mu} J_{\mu}\right)_{\mathrm{anom}}=(D-2)\left(\partial_{\nu} \phi\right) \gamma^{\nu} \psi=-(D-2) \chi \gamma^{\nu} \partial_{\nu} \psi=i(D-2) \chi \mathcal{W}^{\prime \prime}(\phi+\chi) \Psi
$$

where an integration by parts has been carried out, and a total derivative term is omitted (on dimensional grounds it vanishes in the limit $D=2$ ). We also used the equation of motion for the $\psi$ field. The quantum field $\chi$ then forms a loop and we get for the anomaly,

$$
\begin{aligned}
\left(\gamma^{\mu} J_{\mu}\right)_{\mathrm{anom}} & =i(D-2)\left\langle 0\left|\chi^{2}\right| 0\right\rangle \mathcal{W}^{\prime \prime \prime}(\phi) \psi=-(D-2) \int \frac{\mathrm{d}^{D} p}{(2 \pi)^{D}} \frac{1}{p^{2}-m^{2}} \mathcal{W}^{\prime \prime \prime}(\phi) \psi \\
& =\frac{i}{2 \pi} \mathcal{W}^{\prime \prime \prime}(\phi) \psi .
\end{aligned}
$$

The supertransformation of the anomalous term in $\gamma^{\mu} J_{\mu}$ is

$$
\frac{1}{2}\left\{\left(\gamma^{\mu} J_{\mu}\right)_{\mathrm{anom}}, \bar{Q}\right\}=\left(\frac{1}{8 \pi} \mathcal{W}^{\prime \prime \prime \prime} \bar{\psi} \psi-\frac{1}{4 \pi} \mathcal{W}^{\prime \prime \prime} F\right)+i \gamma_{\mu} \gamma^{5} \epsilon^{\mu \nu} \partial_{\nu}\left(\frac{1}{4 \pi} \mathcal{W}^{\prime \prime}\right)
$$

The first term on the right-hand side is the anomaly in the trace of the energymomentum tensor, the second term represents the anomaly in the topological current, the corrected current has the form

$$
\zeta^{\mu}=\epsilon^{\mu \nu} \partial_{\nu}\left(\mathcal{W}+\frac{1}{4 \pi} \mathcal{W}^{\prime \prime}\right)
$$

Consequently, the operator of the central charge

$$
\mathcal{Z}=\left(\mathcal{W}+\frac{1}{4 \pi} \mathcal{W}^{\prime \prime}\right)_{z=+\infty}-\left(\mathcal{W}+\frac{1}{4 \pi} \mathcal{W}^{\prime \prime}\right)_{z=-\infty}
$$

is the difference of $\mathcal{W}+\left(\mathcal{W}^{\prime \prime} / 4 \pi\right)$ at $z= \pm \infty$. Other anomalies in the supermultiplet together with their classical counterparts have the following form,

$$
\begin{aligned}
\gamma^{\mu} J_{\mu} & =2 i\left(\mathcal{W}^{\prime}+\frac{\mathcal{W}^{\prime \prime \prime}}{4 \pi}\right) \psi \\
\vartheta_{\mu}^{\mu} & =-F\left(\mathcal{W}^{\prime}+\frac{\mathcal{W}^{\prime \prime \prime}}{4 \pi}\right)+\frac{1}{2}\left(\mathcal{W}^{\prime \prime}+\frac{\mathcal{W}^{\prime \prime \prime \prime}}{4 \pi}\right) \bar{\psi} \psi .
\end{aligned}
$$

The physical meaning of the anomalous term in the central charge becomes quite apparent from the consideration above. The supersymmetry relates the anomaly in the topological current with a response of the system to the scale transformations,

$$
x \rightarrow(1-\rho) x, \quad \theta \rightarrow(1-(\rho / 2)) \theta, \Phi \rightarrow \Phi .
$$

The kinetic term of the action (2.5) is invariant. The non-invariance of the classical superpotential term is associated with $\mathcal{W}$. This is not the end of the story, however. 
To reveal the anomaly it is convenient, simultaneously with (3.19), to rescale the parameters in the superpotential in accordance with their canonical dimensions. For instance, in the SPM model $m \rightarrow(1+\rho) m, \lambda \rightarrow(1+\rho) \lambda$, and in the SSG model $m \rightarrow(1+\rho) m, v \rightarrow v$. Then,

$$
\mathcal{W} \rightarrow(1+\rho) \mathcal{W}
$$

and the classical action is invariant (the canonical dimension of $\mathcal{W}$ in two dimensions is unity). The quantum action is not invariant, due to the occurrence of a new dimensional parameter, the ultraviolet regulator mass, see e.g. Eq. (4.15). As it follows from Eq. (4.15), the non-invariant contribution is $\mathcal{W}^{\prime \prime} / 4 \pi$. This term gives rise to the anomaly in the central charge density.

It is instructive to compare the above pattern with the anomaly in the central charge in the four-dimensional Wess-Zumino models, discussed previously in the literature [18. In four dimensions, the central charge is proportional to the jump in

$$
3 \mathcal{W}-\sum_{i} \Phi_{i} \frac{\partial \mathcal{W}}{\partial \Phi_{i}}+\frac{1}{8} \sum_{i} \gamma_{i} \bar{D}^{2}\left(\bar{\Phi}_{i} \Phi_{i}\right),
$$

where the summation runs over all chiral superfields $\Phi_{i}$ on which the given Wess-Zumino model is built. Here $\gamma_{i}$ is the anomalous dimension of the field $\Phi_{i}$. The first two terms are purely classical. The coefficient 3 in front of $\mathcal{W}$ is due to the fact that the canonical dimension of the superpotential in the four-dimensional Wess-Zumino model is three. The second term reflects the canonical dimension of the superfield $\Phi$ which is equal to unity in four dimensions. (This is in contrast to two dimensions, where the canonical dimension of $\Phi$ vanishes.) The first two terms in Eq. (3.21), combined together, vanish for purely cubic superpotentials. The reason is quite clear - if the superpotential in the four-dimensional Wess-Zumino model is a cubic polynomial, such a model is classically scale invariant, so the classical part in the central charge must also vanish.

The last term in Eq. (3.21) is the quantum anomaly. It reflects the fact that the field $\Phi$ in loops acquires an anomalous dimension, through a logarithmic renormalization of the kinetic term. In fact, due to the non-renormalization theorem [20], the $Z$ factor for the kinetic term is the only renormalization occurring in the theory. Hence, the term proportional to the anomalous dimensions $\gamma_{i}$ is the only anomalous term. In two dimensions, the kinetic term receives no logarithmically divergent renormalizations, and the anomalous dimension of $\Phi$ vanishes. However, unlike four dimensions, the superpotential itself gets renormalized. This is the origin of the $\mathcal{W}^{\prime \prime} / 4 \pi$ anomalous term in Eq. (3.16) in two dimensions. In four dimensions the anomalous term is a full superderivative, its expectation value over the supersymmetric vacuum vanishes automatically. Therefore, it plays no role in the computation of the central charge for the domain walls [18]. For the very same reason, there is no anomalous correction in the central charge in the two-dimensional soliton problems with $\mathcal{N}=2$. At the same time, the expectation value of the $\mathcal{W}^{\prime \prime}$ anomalous term in two dimensions by no means vanishes. The contribution of the anomaly to the central charge (and, hence, to the mass of the BPS saturated solitons) is absolutely essential for the overall self-consistency of the analysis. 


\subsection{Representations of the centrally extended algebra}

Let us consider the representation of the algebra (3.7) in the soliton sector. In the rest frame, where $P_{z}=0$, we have

$$
Q_{1}^{2}=H+\mathcal{Z}, \quad Q_{2}^{2}=H-\mathcal{Z} .
$$

The supermultiplet consists of two degenerate states: the bosonic $\mid$ sol $\left._{b}\right\rangle$ and fermionic $\mid$ sol $\left._{f}\right\rangle$ kinks. I The supercharge $Q_{1}$ connects them,

$$
Q_{1}\left|\operatorname{sol}_{b}\right\rangle=\sqrt{2 M}\left|\operatorname{sol}_{f}\right\rangle, \quad Q_{1}\left|\operatorname{sol}_{f}\right\rangle=\sqrt{2 M}\left|\operatorname{sol}_{b}\right\rangle .
$$

The second supercharge $Q_{2}$ annihilates both soliton states,

$$
Q_{2}|\mathrm{sol}\rangle=0 \text {. }
$$

This equation is the operator form of the BPS saturation condition. Previously we discussed both features, Eq. (3.23) and Eq. (3.24), at the level of the classical solution.

Note, that an analogue of the condition (3.24) in four dimensions is related to multiplet shortening. This multiplet shortening is possible only if there is a supercharge, which similarly to $Q_{2}$, annihilates some states, as in Eq. (3.24), and its square is related to $H-\mathcal{Z}$, as in Eq. (3.22). In fact, the multiplet shortening is nothing but a signature (sufficient condition) of the existence of an invariant subalgebra. Although we do not have multiplet shortening in two dimensions, the invariant subalgebra formed by $Q_{2}$ and $Q_{2}^{2}$ still exists. The relation (3.24) is enough to guarantee the absence of quantum corrections to $\langle\operatorname{sol}|H-\mathcal{Z}| \operatorname{sol}\rangle$.

Can the soliton become non-saturated after the quantum corrections are switched on? The answer is no. In any supersymmetric theory, if $Q_{2}$ annihilates a state at the tree level, it will continue to do so to any finite order if the coupling constant is small (for a review see e.g. 25]). The proof is based on the fact that the number of degenerate states in the multiplet cannot be increased from two to four in the weak coupling regime. Indeed, if both supercharges, $Q_{1}$ and $Q_{2}$, acted nontrivially one could construct two fermion states, $Q_{1}\left|\mathrm{sol}_{b}\right\rangle$ and $Q_{2}\left|\mathrm{sol}_{b}\right\rangle$. Then the second bosonic state would be obtained as $Q_{1} Q_{2}\left|\operatorname{sol}_{b}\right\rangle$. Since at the classical level there is no extra pair of degenerate states it cannot appear in perturbation theory. Thus, the soliton is protected from losing its saturated nature.

The condition of BPS saturation (3.24) implies that

$$
\langle\mathrm{sol}|H-\mathcal{Z}| \mathrm{sol}\rangle=0
$$

is valid to any order in perturbation theory. In a sense, $H-\mathcal{Z}$ can be viewed as a Hamiltonian for the problem with the boundary effects taken into consideration. Then Eq. (3.25) expresses the complete cancellation of the quantum corrections between bosons and fermions. It relates the soliton mass to the central charge,

$$
M \equiv\langle\operatorname{sol}|H| \operatorname{sol}\rangle=\langle\operatorname{sol}|\mathcal{Z}| \mathrm{sol}\rangle
$$

\footnotetext{
${ }^{2}$ Actually, the supermultiplet is reducible: the states $\left(\left|\operatorname{sol}_{b}\right\rangle \pm\left|\operatorname{sol}_{f}\right\rangle\right) / \sqrt{2}$ present two one-dimensional representations of superalgebra.
} 


\subsection{Ultraviolet regularization by higher derivatives}

We use higher derivatives to regularize the theory in the ultraviolet. The main advantage of the method is the explicit preservation of supersymmetry at every step. To simplify the construction of the canonical formalism we introduce only spatial derivatives for the regularization, the Lorentz noncovariance of the procedure does not lead to a problem.

In regularized form the action (2.5) becomes

$$
S=i \int \mathrm{d}^{2} \theta \mathrm{d}^{2} x\left\{\frac{1}{4} \bar{D}_{\alpha} \Phi\left(1-\frac{\partial_{z}^{2}}{M_{r}^{2}}\right) D_{\alpha} \Phi+\mathcal{W}(\Phi)\right\},
$$

where $M_{r}$ is the regulator mass. In the component form we arrive at the following Lagrangian

$$
\begin{aligned}
\mathcal{L}= & \frac{1}{2}\left\{\partial_{\mu} \phi\left(1-\frac{\partial_{z}^{2}}{M_{r}^{2}}\right) \partial^{\mu} \phi+\bar{\psi}\left(1-\frac{\partial_{z}^{2}}{M_{r}^{2}}\right) i \not \partial \psi+F\left(1-\frac{\partial_{z}^{2}}{M_{r}^{2}}\right) F\right. \\
& \left.+2 \mathcal{W}^{\prime}(\phi) F-\mathcal{W}^{\prime \prime}(\phi) \bar{\psi} \psi\right\}
\end{aligned}
$$

The auxiliary field $F$ can be eliminated from the Lagrangian by virtue of the following formula:

$$
F=-\left(1-\frac{\partial_{z}^{2}}{M_{r}^{2}}\right)^{-1} \mathcal{W}^{\prime}
$$

In perturbation theory near the flat vacuum the bosonic and fermionic propagators are

$$
\begin{array}{cc}
\frac{M_{r}^{2}}{M_{r}^{2}+p_{z}^{2}} \frac{1}{p^{2}-\left(\mathcal{W}_{0}^{\prime \prime}\right)^{2}\left[1+\left(p_{z}^{2} / M_{r}^{2}\right)\right]^{-2}}, & \text { (bosonic) } \\
\frac{M_{r}^{2}}{M_{r}^{2}+p_{z}^{2}} \frac{1}{\not p-\mathcal{W}_{0}^{\prime \prime}\left[1+\left(p_{z}^{2} / M_{r}^{2}\right)\right]^{-1}}, & \text { (fermionic) }
\end{array}
$$

where $\mathcal{W}_{0}^{\prime \prime}$ is the second derivative evaluated at the vacuum value of the field $\phi$. The regularized propagators acquire an extra factor $M_{r}^{2} /\left(M_{r}^{2}+p_{z}^{2}\right)$ making the integrals over $p$ convergent. Strictly speaking, for the quadratically divergent integrals a higher power of the same factor is needed - one can achieve this simply by increasing the power of $\left(1-\partial_{z}^{2} / M_{r}^{2}\right)$ in Eq. (3.27).

Proceeding with canonical quantization in the usual way we get the canonical commutation relations:

$$
\begin{array}{r}
{\left[\phi(t, z),\left(1-\frac{\partial_{z^{\prime}}^{2}}{M_{r}^{2}}\right) \dot{\phi}\left(t, z^{\prime}\right)\right]=i \delta\left(z-z^{\prime}\right),} \\
\left\{\psi_{\alpha}(t, z),\left(1-\frac{\partial_{z^{\prime}}^{2}}{M_{r}^{2}}\right) \bar{\psi}_{\beta}\left(t, z^{\prime}\right)\right\}=\left(\gamma^{0}\right)_{\alpha \beta} \delta\left(z-z^{\prime}\right),
\end{array}
$$


and the regularized Hamiltonian density $\mathcal{H}$

$$
\begin{aligned}
\mathcal{H} & =\dot{\phi}\left(1-\frac{\partial_{z}^{2}}{M_{r}^{2}}\right) \dot{\phi}+\frac{1}{2}\left[\left(1-\frac{\partial_{z}^{2}}{M_{r}^{2}}\right) \bar{\psi}\right] \dot{\psi}-\mathcal{L} \\
& =\frac{1}{2}\left\{\dot{\phi}\left(1-\frac{\partial_{z}^{2}}{M_{r}^{2}}\right) \dot{\phi}+\partial_{z} \phi\left(1-\frac{\partial_{z}^{2}}{M_{r}^{2}}\right) \partial_{z} \phi-\mathcal{W}^{\prime} F-i \bar{\psi}\left(1-\frac{\partial_{z}^{2}}{M_{r}^{2}}\right) \gamma^{1} \psi+\mathcal{W}^{\prime \prime} \bar{\psi} \psi\right\}
\end{aligned}
$$

It is also simple to construct the regularized conserved supercurrent,

$$
J^{\mu}=\left[\left(1-\frac{\partial_{z}^{2}}{M_{r}^{2}}\right)(\not \partial \phi+i F)\right] \gamma^{\mu} \psi+\frac{1}{M_{r}^{2}} \delta_{1}^{\mu}\left\{-\partial_{z}(\not \partial \phi \not \partial \psi)+i F \partial_{z} \not \partial \psi-i\left(\partial_{z} F\right) \not \partial \psi\right\} .
$$

Using the canonical commutation relations presented above one can calculate the commutator of this regularized current with the regularized supercharge. This is a relatively straightforward although tedious exercise. In this way we get regularized expressions for the energy-momentum tensor $\vartheta_{\mu \nu}$ and the topological current $\zeta_{\mu}$. We will analyze them to get the anomaly in the next subsection.

\subsection{Calculation of the anomaly by higher-derivative regular- ization}

The particular anticommutator which determines the regularized topological current $\zeta^{\mu}$ is

$$
\zeta^{\mu}=-\frac{i}{4} \operatorname{Tr}\left(\left\{J^{\mu}, \bar{Q}\right\} \gamma^{5}\right)
$$

where $J^{\mu}$ is presented in Eq. (3.33). The complete expression is rather cumbersome, therefore we will limit ourselves to the time component $\zeta^{0}$ which is sufficient for extracting the anomaly,

$$
\zeta^{0}=\partial_{z}\left\{\mathcal{W}+\frac{1}{2 M_{r}^{2}}\left[\left(\partial_{z} \phi\right) \partial_{z} F-\left(\partial_{z}^{2} \phi\right) F\right]\right\},
$$

where $F$ is given in Eq. (3.29). The term $\partial_{z} \mathcal{W}$ on the right-hand side is the classical topological charge density. The additional terms are due to the regularization, formally they vanish as $1 / M_{r}^{2}$ in the limit of the large regulator mass. However, the loop integration yields $M_{r}^{2}$ in the numerator, so a finite additional term survives.

Using the background field decomposition (3.12), $\phi \longrightarrow \phi_{0}+\chi$, we expand the right-hand side of Eq. (3.35) up to the second order in the quantum field $\chi$ and keep only those terms which survive in the limit $M_{r} \rightarrow \infty$,

$$
\frac{1}{2 M_{r}^{2}}\left[\left(\partial_{z} \phi\right) \partial_{z} F-\left(\partial_{z}^{2} \phi\right) F\right]=\frac{1}{M_{r}^{2}} \mathcal{W}^{\prime \prime}\left\langle\partial_{z} \chi\left(1-\frac{\partial_{z}^{2}}{M_{r}^{2}}\right)^{-1} \partial_{z} \chi\right\rangle .
$$

Strictly speaking, the Green's function of the quantum field $\chi$ depends on the background $\phi_{0}$; see Eq. (3.30). This dependence drops out, however, and the result for the $\chi$ 
loop does not depend on the choice of the background field $\phi_{0}$ since the loop integral is saturated in the domain of virtual momenta $p_{z} \sim M_{r}$. Other domains are irrelevant in the limit $M_{r} \rightarrow \infty$. Thus we can use the bosonic propagator from Eq. (3.30), neglecting the $\left(\mathcal{W}_{0}^{\prime \prime}\right)^{2}$ term in its denominator,

$$
\left\langle\partial_{z} \chi\left(1-\frac{\partial_{z}^{2}}{M_{r}^{2}}\right)^{-1} \partial_{z} \chi\right\rangle=i \int \frac{\mathrm{d}^{2} p}{(2 \pi)^{2}} p_{z}^{2} \frac{1}{p^{2}\left[1+\left(p_{z}^{2} / M_{r}^{2}\right)\right]^{2}}=\frac{M_{r}^{2}}{4 \pi} .
$$

As a result, combining Eqs. (3.35), (3.36), (3.37), we arrive at

$$
\zeta^{0}=\partial_{z}\left\{\mathcal{W}+\frac{\mathcal{W}^{\prime \prime}}{4 \pi}\right\}
$$

which exactly coincides with Eq. (3.16) in the time component. Dimensional regularization and that by higher derivatives lead to one and the same expression for the anomaly in the central charge.

Instead of computing the commutator (3.34) we can suggest an alternative procedure leading directly to Eq. (3.35). To this end it is enough to note that the regularized Hamiltonian density given by Eq. (3.32) can be identically rewritten as follows

$$
\begin{aligned}
\mathcal{H}= & \zeta^{0}+\frac{1}{2}\left\{\dot{\phi}\left(1-\frac{\partial_{z}^{2}}{M_{r}^{2}}\right) \dot{\phi}+\left(\partial_{z} \phi+F\right)\left(1-\frac{\partial_{z}^{2}}{M_{r}^{2}}\right)\left(\partial_{z} \phi+F\right)\right. \\
& \left.-i \bar{\psi}\left(1-\frac{\partial_{z}^{2}}{M_{r}^{2}}\right) \gamma^{1} \psi+\mathcal{W}^{\prime \prime} \bar{\psi} \psi\right\},
\end{aligned}
$$

where the topological charge density $\zeta^{0}$ is the same as in Eq. (3.35) and $F$ is defined by Eq. (3.29).

In the subsequent sections, the very same anomaly will be confirmed, additionally, by two alternative analyses: of the effective one-loop superpotential; and models with an extra superfield. The extra superfield introduced in the framework of softly broken extended supersymmetry serves as a Lorentz-invariant regulator when its mass is taken to be large.

\subsection{One-loop nature of the anomaly}

The anomalous term $\mathcal{W}^{\prime \prime} / 4 \pi$ was obtained at one loop. A natural question is what happens at higher loop orders. In this short subsection we will argue that the expression (3.16) is exact - no higher loop corrections emerge. This should be understood as an operator relation.

The anomaly comes from the ultraviolet range, as we have demonstrated by explicit calculations above. In this range one can consider the superpotential term in the action (2.5) as a perturbation to the first (kinetic) term, which describes massless free fields. The superpotential $\mathcal{W}$ is proportional to the mass $m$, and $m / M_{r}$ is our small parameter. (In the polynomial model the coupling constant $\lambda \sim m v$.) 
Consider, for instance, the operator $\gamma^{\mu} J_{\mu}$. At zeroth order in $\mathcal{W}$ this operator vanishes, in the first order in $\mathcal{W}$ it is proportional to $\mathcal{W}$ (more precisely, $\mathcal{W}$ and its derivatives). All higher loops, i.e. higher orders in $\mathcal{W}$, will result in an extra power of $m / M_{r}$ as far as contributions from ultraviolet domain are concerned. Thus, the first loop is the only one which produces the anomaly.

Does this mean that if one calculates the Feynman graphs for $\gamma^{\mu} J_{\mu}$ the only graph that contributes is one loop? The answer is no. Certainly, multi-loop contributions are present too, but they all represent the matrix element of the operator on the righthand side of Eq. (3.18). The fact that all nonvanishing multi-loop corrections are of infrared origin and, thus, must be referred to the matrix element of the appropriate operator, is obvious on dimensional grounds. Thus, the situation with the anomaly in two-dimensional models under discussion is a perfect parallel to that in SUSY gluodynamics in four dimensions where the three "geometric" anomalies, considered as operator relations, are one-loop exact; all higher-order corrections come as matrix elements of the operator $\operatorname{Tr} W^{2}$ in the given background field [26].

Confirmations of the one-loop nature of the anomaly are provided by calculations in the SSG model presented in Sec. 7 .

\subsection{Infrared regularization and fermion-boson cancellations in local quantities}

To discretize the spectrum of modes let us put the system in a large box, i.e. impose boundary conditions at $z= \pm L / 2$. It is convenient to choose them in a form which is compatible with the residual supersymmetry $(2.20)$. The boundary conditions we are imposing are

$$
\begin{aligned}
& \left.\left(\partial_{z} \phi+F\right)\right|_{z= \pm L / 2}=0, \\
& \left.\left(\partial_{z}+F^{\prime}\right) \psi_{2}\right|_{z= \pm L / 2}=0, \\
& \left.\psi_{1}\right|_{z= \pm L / 2}=0 .
\end{aligned}
$$

It is easy to verify the invariance of these conditions under transformations (2.20). They are also consistent with the classical solutions, both for the flat vacuum and for the kink. In particular, the soliton solution $\phi_{0}$ satisfies to $\partial_{z} \phi+F=0$ everywhere, and boundary conditions (3.40) do not deform it; we can place the kink in the box.

In Sec. 3.3 the exact equality between the soliton mass and the expectation value of the central charge was discussed. Here we will consider a stronger statement, namely the exact equality between the energy density inside the soliton and the expectation value of the central charge density. This equality is valid to any order in perturbation theory and holds locally, for any $z$.

Let us use the expression (3.39) for the energy density $\mathcal{H}$ in the following form,

$$
\mathcal{H}=\zeta^{0}+\frac{1}{2}\left\{(\dot{\phi})^{2}+\left(\partial_{z} \phi+F\right)^{2}+i \psi_{2}\left(\partial_{z}-F^{\prime}\right) \psi_{1}+i \psi_{1}\left(\partial_{z}+F^{\prime}\right) \psi_{2}\right\},
$$


where $\psi_{1,2}$ are the components of the fermion field $\psi_{\alpha}$. We have omitted for simplicity the $1 / M_{r}^{2}$ terms on the right-hand side as they do not change the consideration. Equation (3.41) is the exact operator form. In the classical limit, where $\psi=0$, the expression is positive-definite. Its minimum is achieved provided the field $\phi$ is chosen to be time-independent and to satisfy the BPS condition $\partial_{z} \phi+F=0$. Then the soliton mass is given by the integral over the first term in Eq. (3.41), i.e. by the central charge $\mathcal{Z}$. But now we want to analyze the relation (3.41) in its local form.

For the classical kink solution the difference $\mathcal{H}-\zeta^{0}$ obviously vanishes at any $z$. This vanishing persists at the quantum level,

$$
\left\langle\operatorname{sol}\left|\mathcal{H}(x)-\zeta^{0}(x)\right| \text { sol }\right\rangle=0 .
$$

Indeed, the operator $\mathcal{H}(x)-\zeta^{0}(x)$ can be presented as the anticommutator 10

$$
\left\{Q_{2}, J_{2}^{0}(x)\right\}=\mathcal{H}(x)-\zeta^{0}(x)
$$

which vanishes upon averaging, since $Q_{2} \mid$ sol $\rangle=0$. As a result, not only do the quantum corrections to the kink mass coincide with those for the central charge, but the energy density distribution coincides with the central charge density to all orders.

It is instructive to check in more detail how the cancellation works in the one-loop approximation. To this end we expand the bosonic field $\phi$ near the classical soliton background $\phi_{0}$,

$$
\phi=\phi_{0}+\chi .
$$

In the quadratic in the quantum fields $\chi$ approximation th difference $\mathcal{H}-\zeta^{0}$ takes the following form:

$$
\left[\mathcal{H}-\zeta^{0}\right]_{\text {quad }}=\frac{1}{2}\left\{\dot{\chi}^{2}+\left[\left(\partial_{z}+F^{\prime}\right) \chi\right]^{2}+i \psi_{2}\left(\partial_{z}-F^{\prime}\right) \psi_{1}+i \psi_{1}\left(\partial_{z}+F^{\prime}\right) \psi_{2}\right\}
$$

where $F^{\prime}$ is evaluated at $\phi=\phi_{0}$ and the subscript "quad" denotes the quadratic approximation. We recall that the prime denotes differentiation over $\phi$,

$$
F^{\prime} \equiv \frac{\mathrm{d} F}{\mathrm{~d} \phi}=-\frac{\mathrm{d}^{2} \mathcal{W}}{\mathrm{d} \phi^{2}}
$$

From this expression one can see that a convenient basis for the mode expansion of $\chi$ and $\psi_{2}$ is given by the eigenfunctions $\chi_{n}(z)$ of the Hermitian differential operator $L_{2}$,

$$
L_{2} \chi_{n}(z)=\omega_{n}^{2} \chi_{n}(z), \quad L_{2}=P^{\dagger} P,
$$

where the operators $P$ and $P^{\dagger}$ defined as

$$
P=\partial_{z}+F^{\prime}, \quad P^{\dagger}=-\partial_{z}+F^{\prime}
$$

are Hermitian conjugates. The boundary conditions for the modes in the box follow from Eq. (3.40),

$$
\left(\partial_{z}+F^{\prime}\right) \chi_{n}(z= \pm L / 2)=0 .
$$


As for the basis of the mode expansion for $\psi_{1}$, it is formed by the eigenmodes of the operator $\tilde{L}_{2}$,

$$
\tilde{L}_{2} \tilde{\chi}_{n}(z)=\omega_{n}^{2} \tilde{\chi}_{n}(z), \quad \tilde{L}_{2}=P P^{\dagger},
$$

with the boundary conditions

$$
\tilde{\chi}_{n}(z= \pm L / 2)=0 \text {. }
$$

With boundary conditions (3.48) and (3.50) all eigenvalues of the operators (3.46) and (3.49) are the same, with the exception of the zero mode $\chi_{0} \propto \mathrm{d} \phi_{0}(z) / \mathrm{d} z$ in Eq. (3.46). The operator (3.49) has no zero mode. Moreover, the eigenfunctions $\chi_{n}$ and $\tilde{\chi}_{n}$ are algebraically related,

$$
\tilde{\chi}_{n}=\frac{1}{\omega_{n}} P \chi_{n}, \quad \chi_{n}=\frac{1}{\omega_{n}} P^{\dagger} \tilde{\chi}_{n} .
$$

The expansion in eigenmodes has the form,

$$
\begin{aligned}
& \chi(x)=\sum_{n \neq 0} b_{n}(t) \chi_{n}(z), \quad \psi_{2}(x)=\sum_{n \neq 0} \eta_{n}(t) \chi_{n}(z) \\
& \psi_{1}(x)=\sum_{n \neq 0} \xi_{n}(t) \tilde{\chi}_{n}(z)
\end{aligned}
$$

Note that the summation does not include the zero mode $\chi_{0}(z)$. This mode is not present in $\psi_{1}$ at all. As for the expansions of $\chi$ and $\psi_{2}$, the inclusion of the zero mode would correspond to a shift in the collective coordinates $z_{0}$ and $\eta$ (see Sec. 2.2). Since we deal with the bosonic kink centered at $z=0$, these shifts must be forbidden.

The coefficients $a_{n}, \eta_{n}$ and $\xi_{n}$ are time-dependent operators. Their equal time commutation relations are determined by the canonical commutators (3.3),

$$
\left[b_{m}, \dot{b}_{n}\right]=i \delta_{m n}, \quad\left\{\eta_{m}, \eta_{n}\right\}=\delta_{m n}, \quad\left\{\xi_{m}, \xi_{n}\right\}=\delta_{m n}
$$

Thus, the mode decomposition reduces dynamics of the system under consideration to quantum mechanics of an infinite set of supersymmetric harmonic oscillators (in higher orders the oscillators become anharmonic). The ground state of the quantum soliton corresponds to setting each oscillator in the set to the ground state.

Constructing the creation and annihilation operators in the standard way we find the following nonvanishing expectations values of the bilinears built from the operators $a_{n}, \eta_{n}$ and $\xi_{n}$ in the ground state:

$$
\left\langle\dot{b}_{n}^{2}\right\rangle_{\mathrm{sol}}=\frac{\omega_{n}}{2}, \quad\left\langle b_{n}^{2}\right\rangle_{\mathrm{sol}}=\frac{1}{2 \omega_{n}}, \quad\left\langle\eta_{n} \xi_{n}\right\rangle_{\mathrm{sol}}=\frac{i}{2} .
$$

The expectation values of other bilinears obviously vanish. Combining Eqs. (3.45), (3.52) and (3.54) we get

$$
\left\langle\operatorname{sol}\left|\left[\mathcal{H}(x)-\zeta^{0}\right]_{\text {quad }}\right| \operatorname{sol}\right\rangle=
$$




$$
\frac{1}{2} \sum_{n \neq 0}\left\{\frac{\omega_{n}}{2} \chi_{n}^{2}+\frac{1}{2 \omega_{n}}\left[\left(\partial_{z}+F^{\prime}\right) \chi_{n}\right]^{2}-\frac{\omega_{n}}{2} \chi_{n}^{2}-\frac{1}{2 \omega_{n}}\left[\left(\partial_{z}+F^{\prime}\right) \chi_{n}\right]^{2}\right\} \equiv 0,
$$

The four terms in the braces in Eq. (3.55) are in one-to-one correspondence with the four terms in Eq. (3.45). Note that in proving the vanishing of the right-hand side we did not perform integrations by parts. The vanishing of the right-hand side of (3.45) demonstrates explicitly the residual supersymmetry (i.e. the conservation of $Q_{2}$ ) at work. The cancellation occurs in the very same manner with a finite regulator mass $M_{r}$ due to the residual supersymmetry.

Equation (3.42) must be considered as a local version of BPS saturation (i.e. conservation of a residual supersymmetry). The nonrenormalization of the expectation value of $\mathcal{H}-\zeta^{0}$ over the soliton is a direct analog of the nonrenormalization theorem in the instanton background leading to the exact $\beta$ functions [16] in supersymmetric gluodynamics. The idea of fermion-boson cancellation of the quantum corrections in local quantities was first formulated in Ref. [10. However, in this work the problem of ultraviolet regularization was ignored. Consequently, the quantum anomaly in the central charge density was lost.

In general, the phenomenon of fermion-boson cancellation takes place for the expectation value of every local operator which can be represented as $\left\{Q_{2}, \mathcal{O}(x)\right\}$ where $\mathcal{O}(x)$ is some fermionic operator. We will use this feature later on to calculate the quantum corrections to the energy distribution and to the mean field in the kink.

\subsection{Explicit expressions for the modes and Green's functions}

In this subsection we present explicit expressions for the modes and Green's functions in the SSG model. A nice feature is that everything is expressible in terms of the modes and Green's functions in the flat vacuum. A similar relation exists for the SPM model, and it is outlined at the very end of the subsection. Green's functions in the soliton background are needed for the calculation of the field and energy profiles at one loop; see Sec. 5 .

Let us consider the SSG model. In the kink background the function $F^{\prime}(z)$ which determines the operators $P$ and $P^{\dagger}$ defined in Eq. (3.47) has the form

$$
F^{\prime}=m \tanh m z,
$$

Consequently, the operators $L_{2}$ and $\tilde{L}_{2}$ which determine the mode decomposition are

$$
L_{2}=P^{\dagger} P=-\partial_{z}^{2}+m^{2}-\frac{2 m^{2}}{\cosh ^{2} m z}
$$

and

$$
\tilde{L}_{2}=P P^{\dagger}=-\partial_{z}^{2}+m^{2} .
$$

The key observation is that the operator $\tilde{L}_{2}$ is a quadratic operator in the flat vacuum, and its eigenmodes are those of free motion,

$$
\tilde{\chi}_{n}=\sin p_{n}\left(z+\frac{L}{2}\right) \text {. }
$$


These solutions satisfy the boundary conditions (3.50) at

$$
p_{n}=\frac{\pi n}{L}, \quad n=1,2,3, \ldots,
$$

with the eigenvalues

$$
\omega_{n}^{2}=m^{2}+p_{n}^{2} .
$$

As discussed in the previous subsection, see Eq. (3.51), all nonzero modes $\chi_{n}$ of the operator $L_{2}$ are algebraically expressible via modes $\tilde{\chi}_{n}$. The two operators, $L_{2}$ and $\tilde{L}_{2}$, form a superpair in the sense of Witten's supersymmetric quantum mechanics [25, 27] (for a pedagogical discussion see [28). All non-zero modes of the operator (3.57) are then trivially calculable by virtue of the algebraic relation (3.51),

$$
\chi_{n}(z)=\frac{1}{\omega_{n}}\left[-\partial_{z}+m \tanh m z\right] \sin \left[p_{n}\left(z+\frac{L}{2}\right)\right] .
$$

A similar relation holds for the Green's function,

$$
\begin{gathered}
G_{\omega}\left(z_{1}, z_{2}\right) \equiv i \sum_{n \neq 0} \frac{\chi_{n}\left(z_{1}\right) \chi_{n}\left(z_{2}\right)}{\omega^{2}-\omega_{n}^{2}+i \varepsilon}: \\
G_{\omega}\left(z_{1}, z_{2}\right)=\frac{1}{\omega^{2}}\left(-\partial_{z_{1}}+m \tanh m z_{1}\right)\left(\partial_{z_{2}}+m \tanh m z_{2}\right)\left[\tilde{G}_{\omega}\left(z_{1}, z_{2}\right)-\tilde{G}_{\omega=0}\left(z_{1}, z_{2}\right)\right],
\end{gathered}
$$

where $\tilde{G}_{\omega}\left(z_{1}, z_{2}\right)$ is the Green's function for the free operator $\tilde{L}_{2}$. In the limit of a large box, $L \rightarrow \infty$, the relevant expression for this Green's function is

$$
\tilde{G}_{\omega}\left(z_{1}, z_{2}\right)=-\frac{i}{2 k} \exp \left(-k\left|z_{1}-z_{2}\right|\right)
$$

with $k=\sqrt{m^{2}-\omega^{2}}$. Note that Eq. (3.64) produces the Green's function orthogonal to the zero mode of the operator $L_{2}$. We will make further use these relations in Sec. 5 and 6 .

In the general case, Witten's superpair of operators has the form (see e.g. [28])

$$
-\partial_{z}^{2}+m^{2}-\frac{K(K-1) m^{2}}{\cosh ^{2} m z} \text { and }-\partial_{z}^{2}+m^{2}-\frac{K(K+1) m^{2}}{\cosh ^{2} m z},
$$

where $K$ is integer. We used this fact above at $K=1$, to relate the eigenmodes in the SSG kink background to those of free motion. We can now repeat the trick to relate the eigenmodes in the SPM kink background to those in the SSG kink background, explicitly written above. Indeed, for the SPM kink,

$$
F^{\prime}(z)=m \tanh \frac{m z}{2}
$$

and the quadratic operator which determines the mode decomposition for the field $\chi$ is

$$
L_{2}=P^{\dagger} P=-\partial_{z}^{2}+m^{2}\left[1-\frac{3}{2} \frac{1}{\cosh (m z / 2)}\right] \quad(\mathrm{SPM}) .
$$


The sister operator is

$$
\tilde{L}_{2}=P P^{\dagger}=-\partial_{z}^{2}+m^{2}\left[1-\frac{1}{2} \frac{1}{\cosh (m z / 2)}\right] \quad(\mathrm{SPM}) .
$$

It is not difficult to see that

$$
\tilde{L}_{2}^{\mathrm{SPM}}(z)=\frac{1}{4} L_{2}^{\mathrm{SSG}}(z / 2)+\frac{3}{4} m^{2}
$$

\subsection{Separating soliton from the boundaries}

We pause here to emphasize one important point concerning calculations with boundary conditions. The relation between the eigenmodes in the two sectors connected by the quantum-mechanical supersymmetry operator $P$ and the exact coincidence of the spectra (with one extra zero mode in the kink sector) only holds when the boundary conditions in the two sectors match each other. Namely, these conditions at $z= \pm L / 2$ should be the same for $P \chi_{n} / \omega_{n}$ in the kink sector as for $\tilde{\chi}_{n}$ in the dual sector (the latter is the free motion sector in the case of the $\mathrm{SG}$ model[1). Under these conditions we found the exact local in $z$ boson-fermion cancellation of the difference between the energy density and that of the topological charge in Eq. (3.55). However in other quantities, e.g. in the density of the topological charge, to be considered in the next section, as well as in non-supersymmetric models (like the SG model discussed in Sect. 6), the local cancellation does not take place. In calculation of such quantities one should clearly realize the need for separating the effects of the soliton background from those introduced by the boundaries. The criterion for the separation is obvious: the effects of the boundaries are localized near the boundaries (at distances set by the mass $m$ and by the regulator parameter $M_{r}$ ) and are carried away to infinity in $z$ in the limit of infinitely large box: $L \rightarrow \infty$, while the effects of the soliton proper stay at finite distances, whose scale is set by the mass $m$. This behavior is illustrated in Fig. 1, where the quadratic average of the quantum fluctuations, $\chi^{2}(z)$, is shown for the SG kink background and for the flat background, as calculated with discrete modes in a finite box with a finite ultraviolet regulator parameter. The spikes near the edges of the box are the discussed artifact of the boundary conditions and are moved to infinity in the limit of an infinite box. The soliton background effect is the difference between the curves at the center of the plot, and it remains fixed in the limit $L \rightarrow \infty$.

Thus the effects of the soliton proper can be separated by considering the system in a large box up to distances well inside the box, i.e. up to $z= \pm(L / 2-\Delta)$, where it is sufficient to choose $\Delta$ such that $L \gg \Delta \gg m^{-1}$. This interval of $z$ where the soliton effects already have approached their asymptotic behavior, but the effects of the boundaries of the box are still not essential, can be refered to as fiducial. In what

\footnotetext{
${ }^{3}$ This is not the procedure used in the standard calculations so far: rather the same (usually periodic) boundary conditions are imposed on $\chi_{n}$ and $\tilde{\chi}_{n}$ (see e.g. in [29]), which introduces a difference in the spectra through a phase shift.
} 


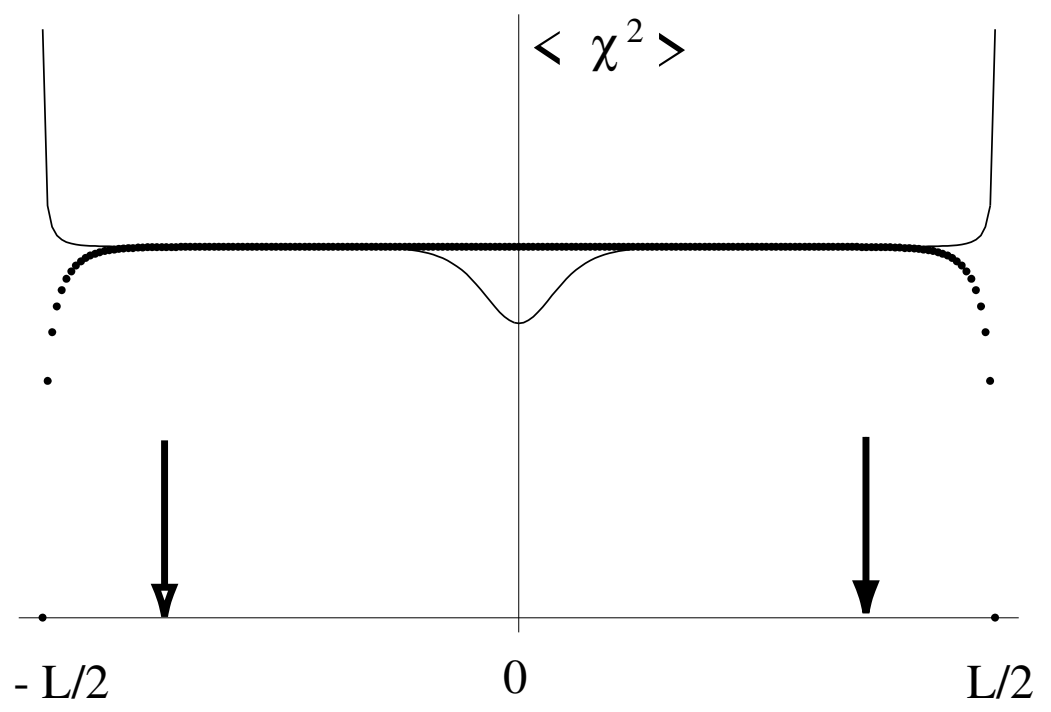

Figure 1: The quadratic average $\chi^{2}(z)$ in a finite box with a finite ultraviolet regulator parameter in the SG kink background (solid line) and for a free field (dots, coalescing into thick line away from the edges of the box). The arrows show the boundaries of the fiducial interval, that encloses the effects of the soliton proper as opposed to those of the boundary conditions.

follows we will imply that only the quantities inside the fiducial interval are considered and the integral quantities are also evaluated over the fiducial interval.

In practice the extraction of the quantities related to the averages over the quantum fluctuations within the fiducial interval is readily done by choosing the appropriate Green's functions for the fields of the fluctuations. Namely, in the limit of the box and the fiducial interval both taken to infinity these Green's functions should be chosen with the Feynman boundary conditions, i.e. they vanish when one of the arguments $z$ goes to either plus or minus infinity, provided that $\omega$ is shifted to the upper complex half-plane. This is the boundary condition under which we have specified the free-motion Green's function in Eq. (3.65)). The discussed above quantum-mechanical supersymmetry then generates the appropriate Green's functions in the soliton sector of the SG model as well as of the SPM model (cf. Eqs. (3.64) and (3.70)), which Green's functions we will be using in the next section. 


\section{The one-loop correction to the kink mass}

\subsection{Central charge and soliton mass at one loop}

Once BPS saturation is proved the actual calculation of quantum corrections to the kink mass becomes a simple job. Indeed, according to Eqs. (3.26) and (3.17)

$$
M=\langle\operatorname{sol}|\mathcal{Z}| \operatorname{sol}\rangle=\left\langle\operatorname{sol}\left|\mathcal{W}+\frac{1}{4 \pi} \mathcal{W}^{\prime \prime}\right| \operatorname{sol}\right\rangle_{z=+\infty}-\left\langle\operatorname{sol}\left|\mathcal{W}+\frac{1}{4 \pi} \mathcal{W}^{\prime \prime}\right| \operatorname{sol}\right\rangle_{z=-\infty}
$$

where the term $\mathcal{W}^{\prime \prime} / 4 \pi$ is due to the anomaly we discussed above in detail. At the oneloop level this term can be substituted by its classical value at $z= \pm \infty$. Additionally, all we need to know is the one-loop quantum corrections to $\langle$ sol $|\mathcal{W}|$ sol $\rangle$ at $z \rightarrow \pm \infty$. These corrections are the same as for the flat vacuum where the standard perturbative expansion can be used.

At $z \rightarrow+\infty$ the classical kink field $\phi_{0}$ goes to the constant $\phi_{*}^{+}$. In the flat vacuum the operators $\phi$ and $\mathcal{W}$ can be presented as

$$
\phi(x)=\phi_{*}^{+}+\chi(x), \quad \mathcal{W}[\phi(x)]=\mathcal{W}_{0}+\frac{1}{2} \mathcal{W}_{0}^{\prime \prime} \chi^{2}(x)+\mathcal{O}\left(\chi^{3}\right),
$$

where $\mathcal{W}_{0}=\mathcal{W}\left[\phi_{*}^{+}\right]$and $\mathcal{W}_{0}^{\prime \prime}=\mathcal{W}^{\prime \prime}\left[\phi_{*}^{+}\right]$are $c$ numbers and $\chi(x)$ is the operator. In the one-loop approximation

$$
\langle\mathcal{W}\rangle_{0}=\mathcal{W}_{0}+\frac{1}{2} \mathcal{W}_{0}^{\prime \prime}\left\langle\chi^{2}(x)\right\rangle_{0}=\mathcal{W}_{0}+\frac{1}{2} \mathcal{W}_{0}^{\prime \prime} G(x, x)
$$

where $G\left(x, x^{\prime}\right)$ is the propagator of the field $\chi$. Using the regularized momentum transform of Eq. (3.30) we get

$$
\left\langle\chi^{2}\right\rangle_{0}=\int \frac{\mathrm{d}^{2} p}{(2 \pi)^{2}} \frac{M_{r}^{2}}{M_{r}^{2}+p_{z}^{2}} \frac{i}{p^{2}-\left(\mathcal{W}_{0}^{\prime \prime}\right)^{2}\left[1+\left(p_{z}^{2} / M_{r}^{2}\right)\right]^{-2}}=\frac{1}{4 \pi} \ln \frac{4 M_{r}^{2}}{\left(\mathcal{W}_{0}^{\prime \prime}\right)^{2}},
$$

where $M_{r}$ is the ultraviolet cut off. Equations (4.3) and (4.4) give the one-loop result for $\langle\mathcal{W}(z \rightarrow+\infty)\rangle$. The result for $\langle\mathcal{W}(z \rightarrow-\infty)\rangle$ is different only by the sign.

Thus, for the kink mass we get

$$
M=2 \mathcal{W}_{0}+\frac{\mathcal{W}_{0}^{\prime \prime}}{4 \pi}\left[\ln \frac{4 M_{r}^{2}}{\left(\mathcal{W}_{0}^{\prime \prime}\right)^{2}}+2\right]
$$

The first term is the classical result for the mass, the one-loop correction is given by the second term, the logarithm in the square brackets arises from the correction to $\langle\mathcal{W}\rangle$ and the additional term 2 is from the anomaly.

It is clear that to make sense of the quantum correction we need to formulate the renormalization procedure. i.e. to fix some observables other than the kink mass. The mass of the light particles proves to be appropriate. It is simple to evaluate the one-loop 
correction to the fermion mass in the SSG model. It is given by the tadpole diagram and can be written as

$$
m_{\text {ren }}=-\left\langle\mathcal{W}^{\prime \prime}\right\rangle_{0}=-\mathcal{W}_{0}^{\prime \prime}-\frac{1}{2} \mathcal{W}_{0}^{\prime \prime \prime \prime}\left\langle\chi^{2}(x)\right\rangle_{0},
$$

where, as above, $\left\langle\chi^{2}\right\rangle_{0}$ appears. At the one-loop level in the SSG model $m_{\text {ren }}$ is both, the on-shell mass, and the renormalized parameter in the superpotential (see the next subsection). Accounting for the fact that in the SSG model $\mathcal{W}=m v^{2} \sin \phi / v$ and $\phi_{*}^{+}=\pi v / 2$ the result (4.5) for the kink mass in terms of $m_{\text {ren }}$ has the form:

$$
M_{\mathrm{SSG}}=2 m_{\mathrm{ren}}\left[v^{2}-\frac{1}{4 \pi}\right]=2 m_{\mathrm{ren}} v^{2}-\frac{m_{\mathrm{ren}}}{2 \pi} .
$$

The logarithm term is absorb by the mass renormalization, what remains is the anomaly term.

In the SPM model the one-loop expression for the on-shell mass is more complicated, it will be obtained in Sec. 5.2. We can introduce, however, an off-shell renormalized mass parameter as follows:

$$
\mu_{\mathrm{ren}}=-\left\langle\mathcal{W}^{\prime \prime}\right\rangle_{0}=2 \lambda \phi_{\mathrm{vac}}=m+2 \lambda\langle\chi\rangle_{0} .
$$

Using the fact that $\left\langle\mathcal{W}^{\prime}\right\rangle_{0}=0$, see Eq. (2.27), we can express $\langle\chi\rangle_{0}$ in terms of $\left\langle\chi^{2}\right\rangle_{0}$ which was determined above,

$$
\langle\chi\rangle_{0}=-\frac{\lambda}{m}\left\langle\chi^{2}\right\rangle_{0}
$$

Then,

$$
M_{\mathrm{SPM}}=\frac{1}{6} \frac{\mu_{\mathrm{ren}}^{3}}{\lambda^{2}}-\frac{\mu_{\mathrm{ren}}}{2 \pi} .
$$

The definitions (4.6) and (4.8) of the renormalized mass parameter are the same as those adopted in Refs. [14, 15], and our results coincide. What then is the difference between our calculations and those of our predecessors?

What was correctly calculated in Refs. [14, 15], as well as in some previous works, was $\langle$ sol $|H|$ sol $\rangle$. The problem was that the result did not match $\langle$ sol $|\mathcal{Z}|$ sol $\rangle$. What we present here is the calculation of $\langle\mathrm{sol}|\mathcal{Z}| \mathrm{sol}\rangle$. We resolve the problem by adding the anomalous term in $\mathcal{Z}$.

The next section presents an effective Lagrangian approach to the calculation of $\mathcal{Z}$ which reproduces the very same anomaly. Moreover, in SPM we suggest another definition of the renormalized (off-shell) mass parameter, which is most natural from the point of view of the effective Lagrangian.

\subsection{Soliton mass from effective superpotential}

A convenient notion to use is the effective superpotential $\mathcal{W}_{\text {eff }}(\phi)$ which appears in the background field approach to perturbation theory. It accounts for the quantum 
corrections in the potential energy which is considered as a function of the background field $\phi_{\text {bck }}$.

Let us calculate one-loop corrections to $\mathcal{W}_{\text {eff }}(\phi)$ choosing as the background constant fields $\phi$ and $F$,

$$
\phi(x)=\phi_{\mathrm{bck}}+\chi(x), \quad F(x)=F_{\mathrm{bck}}+f(x) .
$$

The difference with Eq. (4.2) is that the background field is not a solution of any classical problem and can be chosen arbitrarily. Then the part of the Lagrangian (2.7) quadratic in the quantum fields is

$$
\mathcal{L}^{(2)}=\frac{1}{2}\left\{\partial_{\mu} \chi \partial^{\mu} \chi+f^{2}+2 \mathcal{W}^{\prime \prime} f \chi+F \mathcal{W}^{\prime \prime \prime} \chi^{2}+\bar{\psi} i \not \partial \psi-\mathcal{W}^{\prime \prime} \bar{\psi} \psi\right\}
$$

The one-loop correction to the effective Lagrangian $\mathcal{L}_{\text {eff }}\left(\phi_{\text {bck }}, F_{\text {bck }}\right)$ is defined as

$$
\exp \left\{i \int \mathrm{d}^{2} x \Delta \mathcal{L}_{\text {eff }}\right\}=\int \mathcal{D} \chi \mathcal{D} \psi \exp \left\{i \int \mathrm{d}^{2} x \mathcal{L}^{(2)}\right\}
$$

As is seen from Eq. (2.7) it is sufficient to determine the part of $\Delta \mathcal{L}_{\text {eff }}$ linear in $F_{\text {bck }}$ in the limit $F_{\text {bck }} \rightarrow 0$ to get the one-loop correction to $\mathcal{W}_{\text {eff }}^{\prime}$,

$$
\Delta \mathcal{W}_{\mathrm{eff}}^{\prime}=\frac{1}{2} \mathcal{W}^{\prime \prime \prime}\left\langle\chi^{2}\right\rangle=\left[\frac{\mathcal{W}^{\prime \prime \prime}}{8 \pi} \ln \frac{4 M_{r}^{2}}{\left(\mathcal{W}^{\prime \prime}\right)^{2}}\right]_{\phi_{\mathrm{bck}}} .
$$

As mentioned above, the difference with the previous calculation of $\left\langle\chi^{2}\right\rangle_{0}$ is that $\phi_{\text {bck }}$ is a parameter not equal to its classical value $\phi_{*}^{+}$.

It is easy to construct the one-loop correction to the superpotential $\mathcal{W}_{\text {eff }}$ from its derivative (4.14). The total $\mathcal{W}_{\text {eff }}$ is

$$
\mathcal{W}_{\text {eff }}=\mathcal{W}+\frac{\mathcal{W}^{\prime \prime}}{8 \pi}\left[\ln \frac{4 M_{r}^{2}}{\left(\mathcal{W}^{\prime \prime}\right)^{2}}+2\right]
$$

Note the appearance of the additional 2. This is another alternative way to reproduce the anomaly in the central charge. The origin of this 2 can be traced back to the differentiation of $\ln \phi$ in the superpotential. Thus, within the effective Lagrangian approach the anomaly emerges as an infrared, rather than ultraviolet, effect. This is a usual story - both faces of the anomaly, infrared and ultraviolet, are intertwined.

The condition $\mathcal{W}_{\text {eff }}^{\prime}=0$ defines the shift of the mean vacuum field,

$$
\phi_{\mathrm{vac}}=\langle\operatorname{vac}|\phi| \mathrm{vac}\rangle=\phi_{*}^{+}+\phi_{1}, \quad \phi_{1}=-\frac{1}{8 \pi} \frac{\mathcal{W}_{0}^{\prime \prime \prime}}{\mathcal{W}_{0}^{\prime \prime}} \ln \frac{4 M_{r}^{2}}{\left(\mathcal{W}_{0}^{\prime \prime}\right)^{2}}
$$

At this value of the field

$$
\mathcal{W}_{\text {eff }}\left(\phi_{\text {vac }}\right)=\mathcal{W}_{0}+\frac{\mathcal{W}_{0}^{\prime \prime}}{8 \pi}\left[\ln \frac{4 M_{r}^{2}}{\left(\mathcal{W}_{0}^{\prime \prime}\right)^{2}}+2\right]
$$


The kink mass is defined by the difference between $\mathcal{W}_{\text {eff }}$ at $z= \pm \infty$,

$$
M=2 \mathcal{W}_{\text {eff }}\left(\phi_{\text {vac }}\right)
$$

which coincides with the result (4.5). Thus, we rederive the anomaly from the effective Lagrangian.

Let us stress that the approach presented above is based heavily on supersymmetry. Although we did not use the superfield formalism explicitly it was implicit when we introduced the $F$ component of the background field independent of $\phi$. Had we eliminated the $F$ term from the very beginning the effective Lagrangian would differ by finite (nonlogarithmic) terms.

The effective Lagrangian approach is also convenient for renormalization, all ultraviolet logarithms can be hidden in the parameters of $\mathcal{W}_{\text {eff }}$. In particular, in the polynomial model

$$
\mathcal{W}_{\text {eff }}=\frac{m^{2}}{4 \lambda} \phi-\frac{\lambda}{3} \phi^{3}-\frac{\lambda}{4 \pi} \phi\left[\ln \frac{M_{r}^{2}}{\lambda^{2} \phi^{2}}+2\right], \quad \phi_{\text {vac }}=\frac{m}{2 \lambda}-\frac{\lambda}{4 \pi m} \ln \frac{4 M_{r}^{2}}{m^{2}} .
$$

It is natural to define the renormalized mass $m_{\text {ren }}$ as

$$
m_{\text {ren }}=-\left.\mathcal{W}_{\text {eff }}^{\prime \prime}\right|_{\phi=\phi_{\mathrm{vac}}}=m-\frac{\lambda^{2}}{2 \pi m}\left[\ln \frac{4 M_{r}^{2}}{m^{2}}+2\right] .
$$

In terms of $m_{\text {ren }}$ the expressions for $\mathcal{W}_{\text {eff }}$ and $\phi_{\text {vac }}$ contain no ultraviolet cut off,

$$
\mathcal{W}_{\mathrm{eff}}=\frac{m_{\mathrm{ren}}^{2}}{4 \lambda} \phi-\frac{\lambda}{3} \phi^{3}-\frac{\lambda}{4 \pi} \phi \ln \frac{m_{\mathrm{ren}}^{2}}{4 \lambda^{2} \phi^{2}}, \quad \phi_{\mathrm{vac}}=\frac{m_{\mathrm{ren}}}{2 \lambda}+\frac{\lambda}{2 \pi m_{\mathrm{ren}}},
$$

and the soliton mass is

$$
M_{\mathrm{SPM}}=\frac{1}{6} \frac{m_{\mathrm{ren}}^{3}}{\lambda^{2}} .
$$

This expression superficially looks the same as the classical expression. Of course, one can do things differently, expressing $M$ in terms of $\mu_{\mathrm{ren}}=2 \lambda \phi_{\mathrm{vac}}$ introduced in Eq. (4.8). Then we recover Eq. (4.10). The result for the soliton mass expressed in terms of the on-shell mass $\bar{m}$ of the elementary quanta is given in Eq. (5.26).

In the sine-Gordon model the one-loop effective potential reads as

$$
\mathcal{W}_{\mathrm{eff}}=m v^{2} \sin (\phi / v)-\frac{m \sin (\phi / v)}{8 \pi}\left[\ln \frac{4 M_{r}^{2}}{m^{2} \sin ^{2}(\phi / v)}+2\right]
$$

so that the vacuum field gets no shift t?,

$$
\phi_{\mathrm{vac}}=\phi_{0}=\pi v / 2 .
$$

\footnotetext{
${ }^{4}$ In fact, $\phi_{\text {vac }}$ stays at $\pi v / 2$ to all orders.
} 
while the renormalization of the mass parameter is given by

$$
m_{\mathrm{ren}}=m-\frac{m}{8 \pi v^{2}} \ln \frac{4 M_{r}^{2}}{m^{2}} .
$$

This is identical to Eq. (4.6). Note that in the sine-Gordon model at one loop $m_{\text {ren }}$ in Eq. (4.25) is the same as the on-shell mass $\bar{m}$ of the elementary quanta (see Sec. 5 ). The soliton mass is the same as in Eq. (4.7).

\section{$5 \quad$ Field and energy profile at one loop}

\subsection{General relations for profiles}

The quantum corrections to the kink mass, see Eqs. (4.5), (4.18), are defined by the simple dynamics of the flat vacuum at $z \rightarrow \pm \infty$. Here we will find the one-loop corrections to two local quantities: the mean field and the energy density in the kink. We start with a general superpotential $\mathcal{W}(\Phi)$, and then demonstrate the explicit solution for the polynomial and sine-Gordon models. Similar corrections to the domain wall profile in four dimensions were found previously in non-supersymmetric models in [21, 22] and in supersymmetric theories in [18].

The mean field $\bar{\phi}(z)$ is defined as

$$
\bar{\phi}(z)=\langle\operatorname{sol}|\phi(t, z)| \operatorname{sol}\rangle=\phi_{0}(z)+\phi_{1}(z)
$$

where we denote as $\phi_{1}(z)$ the deviation of the mean field from the classical kink solution $\phi_{0}(z)$. To derive the equation for the mean field let us start from the anticommutator

$$
\left\{\psi_{1}, Q_{2}\right\}=-\left(\partial_{z} \phi+F\right)
$$

where $F$ denotes $-\mathcal{W}^{\prime}(\phi)$. As was discussed in Sec. 3.7 the expectation value of the anticommutator vanishes due to BPS saturation, $Q_{2} \mid$ sol $\rangle=0$. Therefore, we get for the mean field

$$
\frac{\mathrm{d}}{\mathrm{d} z} \bar{\phi}(z)=-\langle\mathrm{sol}|F| \mathrm{sol}\rangle \text {. }
$$

Expanding the operator field $\phi$ near the classical solution,

$$
\phi=\phi_{0}(z)+\phi_{1}(z)+\chi(t, z)
$$

we get from Eq. (5.3)

$$
\frac{\mathrm{d}}{\mathrm{d} z} \phi_{1}(z)=-F^{\prime} \phi_{1}(z)-\frac{1}{2} F^{\prime \prime}\left\langle\chi^{2}(z, t)\right\rangle_{\mathrm{sol}} .
$$

Throughout this section the derivatives of the superpotential with respect to the field $\phi$, denoted by the appropriate number of primes, are evaluated at the classical profile, i.e. 
at $\phi=\phi_{0}(z)$. One should also notice that the quadratic average of the quantum fluctuations $\left\langle\chi^{2}(t, z)\right\rangle$ is in fact time-independent, since it is calculated in the background of a static field configuration.

Let us first consider the behavior of the solution of Eq. (5.5) at the infinities. For the limiting behavior at $z \rightarrow \pm \infty$ we found in Sec. 4, see Eq. (4.16),

$$
\lim _{z \rightarrow \pm \infty} \phi_{1}(z)=-\left.\frac{\mathcal{W}_{0}^{\prime \prime \prime}}{2 \mathcal{W}_{0}^{\prime \prime}}\left\langle\chi^{2}(z, t)\right\rangle\right|_{z \rightarrow \pm \infty}=-\left.\frac{1}{8 \pi} \frac{\mathcal{W}_{0}^{\prime \prime \prime}}{\mathcal{W}_{0}^{\prime \prime}} \ln \frac{4 M_{r}^{2}}{\left(\mathcal{W}_{0}^{\prime \prime}\right)^{2}}\right|_{z \rightarrow \pm \infty}
$$

These limits are consistent with Eq. (5.5), its left-hand side is vanishing at $z \rightarrow \pm \infty$.

Using the expansion (3.52) of $\chi$ in eigenmodes and Eq. (3.54) we find for the quadratic average

$$
\left\langle\chi^{2}(x)\right\rangle_{\mathrm{sol}}=\sum_{n \neq 0} \frac{\chi_{n}^{2}(z)}{2 \omega_{n}} .
$$

The same result can also be presented as

$$
\left\langle\chi^{2}(x)\right\rangle_{\mathrm{sol}}=G(x ; x)
$$

where $G(x ; x)$ is the limit at coinciding space-time points of the Green's function $G\left(x ; x^{\prime}\right)$, satisfying the second order equation

$$
\left[-\partial_{t}^{2}+\left(\partial_{z}-F^{\prime}\right)\left(\partial_{z}+F^{\prime}\right)\right] G\left(x ; x^{\prime}\right)=i \delta\left(t-t^{\prime}\right)\left[\delta\left(z-z^{\prime}\right)-\chi_{0}(z) \chi_{0}\left(z^{\prime}\right)\right] .
$$

The subtraction of $\chi_{0}(z) \chi_{0}\left(z^{\prime}\right)$ in the right-hand side is needed for the exclusion of the zero mode.

Due to the time independence of the background, the Green's function is conveniently expanded in frequency modes:

$$
G\left(t ; z ; t^{\prime}, z^{\prime}\right)=\int_{-\infty}^{+\infty} G_{\omega}\left(z ; z^{\prime}\right) e^{-i \omega\left(t-t^{\prime}\right)} \frac{\mathrm{d} \omega}{2 \pi}
$$

where $G_{\omega}\left(z ; z^{\prime}\right)$ satisfies the one-dimensional equation

$$
\left[\omega^{2}+\left(\partial_{z}-F^{\prime}\right)\left(\partial_{z}+F^{\prime}\right)\right] G_{\omega}\left(z ; z^{\prime}\right)=i\left[\delta\left(z-z^{\prime}\right)-\chi_{0}(z) \chi_{0}\left(z^{\prime}\right)\right]
$$

The solution to this equation for $G_{\omega}\left(z_{1} ; z_{2}\right)$ can be written in terms of the normalized eigenfunctions $\chi_{n}(z)$ and the corresponding eigenvalues $\omega_{n}$ defined in Eq. (3.46) in the standard way,

$$
G_{\omega}\left(z ; z^{\prime}\right)=i \sum_{n \neq 0} \frac{\chi_{n}(z) \chi_{n}\left(z^{\prime}\right)}{\omega^{2}-\omega_{n}^{2}+i \varepsilon}
$$

At coinciding points we get

$$
\left\langle\chi^{2}(x)\right\rangle_{\mathrm{sol}}=G(x ; x)=\int_{-\infty}^{+\infty} G_{\omega}(z ; z) \frac{\mathrm{d} \omega}{2 \pi},
$$


and the integration over $\omega$ reproduces the representation 5.7 ).

In terms of the quantity $\left\langle\chi^{2}(z)\right\rangle$ the solution to the equation (5.5) for the correction to the field profile can be written as

$$
\phi_{1}(z)=-\frac{1}{2} \chi_{0}(z) \int_{0}^{z} \frac{F^{\prime \prime}\left(\phi_{0}(u)\right)\left\langle\chi^{2}(u)\right\rangle}{\chi_{0}(u)} \mathrm{d} u=-\frac{1}{2} F\left(\phi_{0}(z)\right) \int_{0}^{z} \frac{F^{\prime \prime}\left(\phi_{0}(u)\right)\left\langle\chi^{2}(u)\right\rangle}{F\left(\phi_{0}(u)\right)} \mathrm{d} u,
$$

where the last equality makes use of the fact that the translational mode $\chi_{0}$ is proportional to $F$ due to the BPS saturation at the classical level. The lower limit in the integral is the collective coordinate for the soliton center which we have chosen to be at $z=0$.

Once $\left\langle\chi^{2}(x)\right\rangle$ and $\phi_{1}$ are found the quantum correction to the energy distribution in the kink is also fully determined. The quantity

$$
\begin{aligned}
M(z) & =2\left\langle\mathcal{W}(z)+\frac{\mathcal{W}^{\prime \prime}(z)}{4 \pi}\right\rangle_{\mathrm{sol}}=2 \mathcal{W}+\frac{\mathcal{W}^{\prime \prime}}{2 \pi}+2 \mathcal{W}^{\prime} \phi_{1}+\mathcal{W}^{\prime \prime}\left\langle\chi^{2}\right\rangle_{\mathrm{sol}} \\
& =2\left[\mathcal{W}-\frac{F^{\prime}}{4 \pi}-\left(F-\frac{\left(F^{\prime}\right)^{2}}{F^{\prime \prime}}\right) \phi_{1}+\frac{F^{\prime}}{F^{\prime \prime}} \frac{\mathrm{d} \phi_{1}}{\mathrm{~d} z}\right]
\end{aligned}
$$

represents the total energy located in the interval between $-z$ and $z$ (implying that $z>$ $0)$. In the last expression $\mathcal{W}$ and its derivatives over $\phi$ are evaluated at $\phi=\phi_{0}(z)$. In the large $z$ limit $M(z)$ goes to the soliton mass $M$. The energy density is $(1 / 2) \mathrm{d} M(z) / \mathrm{d} z$.

Thus, the one-loop corrections to the mean field and to the energy density are expressed via integrals over the Green's function $G_{\omega}(z ; z)$ defined by Eq. (5.11). These results were obtained for a general superpotential function $\mathcal{W}$. In the special cases of the polynomial and sine-Gordon superpotentials (see Eqs. (2.8) and (2.9)) this Green's

function is known analytically and we will get explicit expressions for the one-loop corrections.

\subsection{Polynomial model}

The partial Green function $G_{\omega}\left(z_{1} ; z_{2}\right)$ is readily constructed by twice applying the relation (3.64) to the free Green's function in Eq. (3.65) (it can also be read off from Refs. 21,22]). At coinciding points $G_{\omega}(z ; z)$ is given by

$$
\begin{aligned}
G_{\omega}(z ; z)= & -\frac{i}{2 \sqrt{m^{2}-\omega^{2}}}\left[1-\frac{1}{\omega^{2}} \frac{3 m^{2}}{4 \cosh ^{4}(m z / 2)}\left(1-\frac{\sqrt{m^{2}-\omega^{2}}}{m}\right)\right. \\
& \left.+\frac{1}{3 m^{2}-4 \omega^{2}} \frac{3 m^{2} \tanh ^{2}(m z / 2)}{\cosh ^{2}(m z / 2)}\right] .
\end{aligned}
$$

Thus, substituting this expression in Eq. (5.13) and performing Euclidean rotation in the integration over $\omega$ (i.e. the substitution $\omega \rightarrow i \omega$ ) one finds for the average over the 
fluctuations

$$
\begin{aligned}
\left\langle\chi^{2}(z)\right\rangle_{\mathrm{sol}} & =\int_{-\infty}^{+\infty} \frac{d \omega}{4 \pi \sqrt{\omega^{2}+m^{2}}}\left[1+\frac{1}{\omega^{2}} \frac{3 m^{2}}{4 \cosh ^{4}(m z / 2)}\left(1-\frac{\sqrt{\omega^{2}+m^{2}}}{m}\right)\right. \\
& \left.+\frac{1}{4 \omega^{2}+3 m^{2}} \frac{3 m^{2} \tanh ^{2}(m z / 2)}{\cosh ^{2}(m z / 2)}\right] \\
& =\frac{1}{4 \pi} \ln \frac{4 M_{r}^{2}}{m^{2}}-\frac{3}{8 \pi} \frac{1}{\cosh ^{4}(m z / 2)}+\frac{1}{4 \sqrt{3}} \frac{\tanh ^{2}(m z / 2)}{\cosh ^{2}(m z / 2)} .
\end{aligned}
$$

It is understood here that the divergent part of the integral is regularized by the ultraviolet regularization procedure described above. Upon substitution in Eq. (5.14) this expression gives the final result for the correction to the profile of the field across the soliton:

$$
\begin{aligned}
& \phi_{1}(z)=-\left.\frac{\lambda^{2}}{2 \pi m}\left(\ln \frac{4 M_{r}^{2}}{m^{2}}+\frac{2 \pi}{\sqrt{3}}\right) \frac{\partial \phi_{0}}{\partial m}\right|_{\lambda}-\left.\frac{\lambda^{3}}{\sqrt{3} m^{2}} \frac{\partial \phi_{0}}{\partial \lambda}\right|_{m} \\
& +\frac{\lambda}{m}\left(\frac{1}{2 \sqrt{3}}+\frac{3}{4 \pi}\right) \frac{\tanh (m z / 2)}{\cosh ^{2}(m z / 2)} .
\end{aligned}
$$

The first two terms in this expression clearly can be absorbed in a redefinition of the parameters $m$ and $\lambda$ in the classical profile of the field. Thus the full one-loop corrected field $\bar{\phi}$ across the soliton can be written as

$$
\bar{\phi} \equiv \phi_{0}(z)+\phi_{1}(z)=\frac{\bar{m}}{\overline{2} \lambda} \tanh (\bar{m} z / 2)+\frac{\bar{\lambda}}{\bar{m}}\left(\frac{1}{2 \sqrt{3}}+\frac{3}{4 \pi}\right) \frac{\tanh (\bar{m} z / 2)}{\cosh ^{2}(\bar{m} z / 2)},
$$

where

$$
\bar{m}=m-\frac{\lambda^{2}}{2 \pi m}\left(\ln \frac{4 M_{r}^{2}}{m^{2}}+\frac{2 \pi}{\sqrt{3}}\right) \text { and } \bar{\lambda}=\lambda-\frac{\lambda^{3}}{\sqrt{3} m^{2}}
$$

are the appropriately renormalized parameters. It should be noted that the mass parameter in this renormalization scheme exactly corresponds to the on-shell mass renormalization. In other words, the physical (pole) renormalized mass of the scalar boson (and, by supersymmetry, of the fermion) is equal to $\bar{m}$, as can be seen from the exponential approach of the profile in Eq. (5.19) to the vacuum value at $z \rightarrow \infty$.

Substituting the result (5.18) for the profile correction into Eq. (5.15) we find the energy $M(z)$ sitting between $-z$ and $z$,

$$
\begin{aligned}
& M(z)=\frac{\bar{m}^{3}}{6 \bar{\lambda}^{2}} \tanh (\bar{m} z / 2)\left(2+\frac{1}{\cosh ^{2}(\bar{m} z / 2)}\right)\left(1+\frac{1}{\sqrt{3}} \frac{\bar{\lambda}^{2}}{\bar{m}^{2}}\right) \\
& -\frac{\bar{m}}{2 \pi} \tanh (\bar{m} z / 2)+\bar{m} \frac{\tanh (\bar{m} z / 2)}{\cosh ^{2}(\bar{m} z / 2)}\left[-\frac{1}{4 \sqrt{3}}+\left(\frac{1}{2 \sqrt{3}}+\frac{3}{4 \pi}\right) \frac{1}{\cosh ^{2}(\bar{m} z / 2)}\right] .
\end{aligned}
$$

The first line is similar to the classical energy distribution up to the transition to renormalized parameters. The correction factor in this line is equivalent to an additional finite renormalization of $\lambda$. 
The qualitative feature which quantum corrections bring in is that $M(z)$ approaches its asymptotic value at large $z$ more slowly. Classically (the first line) it approaches as $\exp (-2 m z)$. The one-loop correction contains $\exp (-m z)$. This can be simply understood in perturbation theory: the energy density operator can only produce one-particle states at the quantum level.

We also find it quite instructive to consider a "kink effective superpotential" $\overline{\mathcal{W}}_{\text {eff }}(\bar{\phi})$, which generates the full one-loop profile of the field, as the solution to an "effective" equation

$$
\frac{\mathrm{d}}{\mathrm{d} z} \bar{\phi}=\overline{\mathcal{W}}_{\text {eff }}^{\prime}(\bar{\phi})
$$

This effective function can readily be found by noticing that the expression for the average $\left\langle\chi^{2}(z)\right\rangle_{\text {sol }}$ given by Eq. (5.17), which enters the quantum BPS equation (5.5), is in fact a fourth power polynomial in the classical solution $\phi_{0}(z)$. Thus the inhomogeneous term in Eq. (5.5) can be written as the derivative of a fifth power polynomial in $\phi$. Hence one arrives at the following expression for $\overline{\mathcal{W}}_{\text {eff }}$

$$
\overline{\mathcal{W}}_{\mathrm{eff}}(\bar{\phi})=\frac{\bar{m}^{2}}{4 \bar{\lambda}} \bar{\phi}-\frac{\bar{\lambda}}{3} \bar{\phi}^{3}+\bar{\lambda}\left(\frac{1}{4 \sqrt{3}}+\frac{3}{8 \pi}\right)\left[\bar{\phi}-\frac{8 \bar{\lambda}^{2}}{3 \bar{m}^{2}} \bar{\phi}^{3}+\frac{16 \bar{\lambda}^{4}}{5 \bar{m}^{4}} \bar{\phi}^{5}\right]
$$

It is important to emphasize that the so defined soliton effective (super)potential $\overline{\mathcal{W}}_{\text {eff }}$ is different from the Coleman-Weinberg type effective (super)potential $\mathcal{W}_{\text {eff }}$ in Eq. (4.15). The latter describes the response of the action to a space-time constant field, and is appropriate for finding the quantum corrections to the vacuum field, while the purpose of the former is to describe the quantum corrections to the soliton profile through the equation (5.22). In fact, the $\overline{\mathcal{W}}_{\text {eff }}$ also describes the correction to the vacuum field as the asymptotic value of the solution at $z \rightarrow \infty$, which perfectly agrees with $\phi_{\text {vac }}$ found from $\mathcal{W}_{\text {eff }}: \overline{\mathcal{W}}_{\text {eff }}^{\prime}\left(\phi_{\text {vac }}\right)=0$. In addition the soliton effective superpotential encodes all information about the detailed behavior of the field profile at finite $z$. In particular, its second derivative gives the on-shell mass of the elementary particles,

$$
\left.\overline{\mathcal{W}}_{\text {eff }}^{\prime \prime}\right|_{\phi=\phi_{\text {vac }}}=-\bar{m}
$$

unlike the mass parameter $m_{\text {ren }}$, calculated in Eq. (4.20) from the curvature of $\mathcal{W}_{\text {eff }}$,

$$
\bar{m}=m_{\mathrm{ren}}+\frac{\lambda^{2}}{\pi m}\left(1-\frac{\pi}{\sqrt{3}}\right) .
$$

Thus, the mass of the kink from Eq. (4.22), being expressed through the on-shell mass parameter $\bar{m}$ reads as

$$
M=\frac{1}{6} \frac{\bar{m}^{3}}{\lambda^{2}}+\frac{\bar{m}}{2 \sqrt{3}}-\frac{\bar{m}}{2 \pi}=\frac{1}{6} \frac{\bar{m}^{3}}{\bar{\lambda}^{2}}+\frac{\bar{m}}{6 \sqrt{3}}-\frac{\bar{m}}{2 \pi} .
$$

The same expression for $M$ appears in Eq. (5.22) as the asymptotic value of $M(z)$ at large $z$. The first two terms in (5.26) are equivalent to the result of Ref. [10] where it 
was obtained within a similar approach. However our result differs by the third term, which arises from the anomalous part of the central charge.

A remarkable property of the soliton effective (super)potential is that it is a finite polynomial of the field, unlike that of the Coleman-Weinberg type. This is due to the phenomenon of "nullification" [21, 22, 30]: the vanishing of all on-shell amplitudes for multiboson production at threshold, starting from a finite number of the produced bosons. The on-shell amplitudes are related to the profile of the soliton field [21, 31].

\subsection{Sine-Gordon model}

Following the same routine as for the polynomial model, we write the expression for the partial Green's function $G_{\omega}(z ; z)$ in the background of the classical soliton profile given by Eq. (2.12),

$$
G_{\omega}(z ; z)=-\frac{i}{2 \sqrt{m^{2}-\omega^{2}}}\left[1-\frac{1}{\omega^{2}} \frac{m^{2}}{\cosh ^{2} m z}\left(1-\frac{\sqrt{m^{2}-\omega^{2}}}{m}\right)\right] .
$$

The average over the quantum fluctuations then takes the form

$$
\begin{aligned}
& \left\langle\chi^{2}(z)\right\rangle_{\mathrm{sol}}=\int_{-\infty}^{+\infty} \frac{d \omega}{4 \pi \sqrt{\omega^{2}+m^{2}}}\left[1+\frac{1}{\omega^{2}} \frac{m^{2}}{\cosh ^{2} m z}\left(1-\frac{\sqrt{\omega^{2}+m^{2}}}{m}\right)\right] \\
& =\frac{1}{4 \pi} \ln \frac{4 M_{r}^{2}}{m^{2}}-\frac{1}{2 \pi} \frac{1}{\cosh ^{2} m z} .
\end{aligned}
$$

Thus, according to Eq. (5.14), the one-loop correction to the soliton profile is given by

$$
\phi_{1}(z)=-\left.\frac{m}{8 \pi v^{2}} \ln \frac{4 M_{r}^{2}}{m^{2}} \frac{\partial \phi_{0}}{\partial m}\right|_{v}+\frac{1}{4 \pi v} \frac{\tanh m z}{\cosh m z} .
$$

Absorbing the first term in this expression into a renormalization of the mass in the classical field profile, one can write the final expression for the full one-loop profile of the soliton field $\bar{\phi}$ as

$$
\bar{\phi}(z)=2 v \arctan [\tanh (\bar{m} z / 2)]+\frac{1}{4 \pi v} \frac{\tanh \bar{m} z}{\cosh \bar{m} z}
$$

where

$$
\bar{m}=m-\frac{m}{8 \pi v^{2}} \ln \frac{4 M_{r}^{2}}{m^{2}}
$$

is the renormalized on-shell mass of the elementary particles in the sine-Gordon model. One can readily notice that in this model at one loop the renormalized pole mass coincides with the one defined from the one-loop effective potential: $m_{\text {ren }}$ in Eq. (4.25). This is no surprise, since in this model at one loop there is no dispersion of the particles' self-energy. (This property, and the relation $\bar{m}=m_{\text {ren }}$ is lost, however, at the two-loop level, see Sec.7.) 
The amount of energy located in the interval between $-z$ and $z$ is readily calculated from Eq. (5.15) and is given by

$$
M(z)=2 \bar{m}\left(v^{2}-\frac{1}{4 \pi}\right) \tanh \bar{m} z+\frac{\bar{m}}{\pi} \frac{\tanh \bar{m} z}{\cosh ^{2} \bar{m} z} .
$$

One can notice that in this case the approach of $M(z)$ to its asymptotic value $M$ is described by terms proportional to $\exp (-2 m z)$, i.e. corresponding to propagation of a two-particle state. This is due to the fact that in the sine-Gordon model the number of particles is conserved modulo 2, so that the energy operator does not couple through quantum loops to a one-particle state.

The soliton effective superpotential is also readily recovered in this model and reads as follows,

$$
\overline{\mathcal{W}}_{\text {eff }}=\bar{m} v^{2} \sin \frac{\bar{\phi}}{v}+\frac{\bar{m}}{4 \pi}\left(\sin \frac{\bar{\phi}}{v}-\frac{1}{3} \sin ^{3} \frac{\bar{\phi}}{v}\right),
$$

and is polynomial in $\sin (\bar{\phi} / v)$.

\section{6 "Supersymmetry" of the non-SUSY sine-Gordon model.}

Here we will discuss a "supersymmetry" in the problem of calculating the soliton mass (at one loop) in the non-supersymmetric sine-Gordon model, whose Hamiltonian is

simply the bosonic part of that of the SSG model. The sine-Gordon Hamiltonian density can be written as

$$
\mathcal{H}=\partial_{z} \mathcal{W}+\frac{1}{2}\left\{\dot{\phi}^{2}+\left(\partial_{z} \phi+F\right)^{2}\right\}
$$

with $\mathcal{W}(\phi)$ given by Eq. (2.9) and $F(\phi)=-\mathcal{W}(\phi)^{\prime}=-m v \cos (\phi / v)$. Since the purpose of this section is mainly illustrative, we will ignore the necessity for ultraviolet regularization. This can be done along the lines of Sec. 3.5. We will not introduce the explicit regularization to avoid cumbersome formulae which could overshadow our key points. One should understand that in doing so we are bound to miss some finite (non-logarithmic) piece in the soliton mass. This flaw could easily be eliminated, if desired.

In order to write the one-loop (quadratic) expression for the energy in terms of the quantum fluctuations $\chi$ over the kink background $\phi_{0}(z)$ (see Eq. (2.12)) one should take into account that, unlike the supersymmetric case, the flat vacuum energy density receives a one-loop renormalization and is to be subtracted. The proper expression is as follows:

$$
\langle\mathcal{H}\rangle_{\mathrm{kink}}-\langle\mathcal{H}\rangle_{\mathrm{vac}}-\partial_{z} \mathcal{W}=\frac{1}{2}\left\{\dot{\chi}^{2}+\left[\left(\partial_{z}+F^{\prime}\right) \chi\right]^{2}-\dot{\tilde{\chi}}^{2}-\left(\partial_{z} \tilde{\chi}\right)^{2}-m^{2} \tilde{\chi}^{2}\right\} .
$$


Here $F^{\prime}=m \tanh m z$ is the background value at $\phi_{0}(z)$, and $\mathcal{W}$ is the profile of $m v^{2} \sin (\phi / v)$ across the soliton (including the one-loop correction). Finally, $\tilde{\chi}$ denotes in this section the field fluctuations over the flat vacuum at $\phi_{0}=\pi v / 2$ (see Sec. 3.8).

We consider the contribution of the quantum fluctuations $\chi$ and $\tilde{\chi}$, explicitly written in Eq. (6.2), by expanding these fields in the appropriate eigenmodes. Namely, the field $\chi$ is expanded in the eigenmodes $\chi_{n}(z)$ of the operator

$$
L_{2}=-\left(\partial_{z}-F^{\prime}\right)\left(\partial_{z}+F^{\prime}\right)=-\partial_{z}^{2}+m^{2}-\frac{2 m^{2}}{\cosh ^{2} m z}
$$

while the corresponding operator for the modes $\tilde{\chi}_{n}$ of the field $\tilde{\chi}$ in the flat vacuum is obviously

$$
\tilde{L}_{2}=-\partial_{z}^{2}+m^{2}
$$

Since the operators $L_{2}$ and $\tilde{L}_{2}$ are related by a quantum-mechanical supersymmetry (see Sec. 3.8), we use here the fact that the spectra of both operators coincide (modulo the zero mode of $L_{2}$ ). We also use the ensuing relations for the eigenmodes. It is seen that the soliton sector can be dubbed "bosonic" while the flat vacuum "fermionic". These two sectors are related by a quantum-mechanical "supercharge" $P=\left(\partial_{z}+F^{\prime}\right)$.

With our boundary conditions, the terms in Eq. (6.2) can be formally f written as follows:

$$
\begin{aligned}
& \left\langle\dot{\chi}^{2}\right\rangle=\sum_{\omega_{n} \neq 0} \frac{\omega_{n}}{2} \chi_{n}^{2}(z), \\
& \left\langle\left[\left(\partial_{z}+F^{\prime}\right) \chi\right]^{2}\right\rangle=\sum_{\omega_{n} \neq 0} \frac{\omega_{n}}{2} \tilde{\chi}_{n}^{2}(z), \\
& \left\langle\dot{\tilde{\chi}}^{2}\right\rangle=\sum_{\omega_{n} \neq 0} \frac{\omega_{n}}{2} \tilde{\chi}_{n}^{2}(z), \\
& \left\langle\left(\partial_{z} \tilde{\chi}\right)^{2}+m^{2} \tilde{\chi}^{2}\right\rangle=\frac{1}{2} \partial_{z}^{2} \sum_{\omega_{n} \neq 0} \frac{\tilde{\chi}_{n}^{2}(z)}{2 \omega_{n}}+\sum_{\omega_{n} \neq 0} \frac{\omega_{n}}{2} \tilde{\chi}_{n}^{2}(z) .
\end{aligned}
$$

Here the relation

$$
P \chi_{n}(z)=\omega_{n} \tilde{\chi}_{n}(z)
$$

is used in the second line. Note also that the total derivatives are explicitly kept. Using these relations, the expression (6.2) for the kink energy density can be formally rearranged as

$$
\langle\mathcal{H}\rangle_{\text {kink }}-\langle\mathcal{H}\rangle_{\text {vac }}-\partial_{z} \mathcal{W}=-\frac{1}{4} \partial_{z}^{2} \sum_{\omega_{n} \neq 0} \frac{\tilde{\chi}_{n}^{2}(z)}{2 \omega_{n}}+\frac{1}{2} \sum_{\omega_{n} \neq 0} \frac{\omega_{n}}{2}\left[\chi_{n}^{2}(z)-\tilde{\chi}_{n}^{2}(z)\right] .
$$

\footnotetext{
${ }^{5}$ As was mentioned, for illustrative purposes we ignore here the problem of the specific ultraviolet regularization of the one-loop terms in the sine-Gordon model. Thus, an analog of the anomalous term $\mathcal{W}^{\prime \prime} / 4 \pi$ in the central charge will be lost. We will focus only on the logarithmically divergent part of the soliton mass renormalization.
} 
It can be noticed, that the integral of each term in the last sum in the right-hand side of the latter equation over the whole box vanishes identically, since the modes in both sectors are normalized to one. However the relevant integral over the fiducial interval, discussed in the Sec. 3.9 is not zero, due to different behavior of the modes at the edges of the bounding boxf. In order to calculate the integral over the latter interval we again use the quantum-mechanical supersymmetry to express the modes $\chi_{n}$ in terms of $\tilde{\chi}_{n}$,

$$
\begin{aligned}
& \chi_{n}^{2}=\frac{1}{\omega_{n}^{2}}\left(P^{\dagger} \tilde{\chi}_{n}\right)^{2}=\frac{1}{\omega_{n}^{2}} \partial_{z}\left[\tilde{\chi}_{n}\left(\partial_{z}-F^{\prime}\right) \tilde{\chi}_{n}\right]+\frac{\tilde{\chi}_{n} P P^{\dagger} \tilde{\chi}_{n}}{\omega_{n}^{2}}= \\
& \frac{1}{\omega_{n}^{2}} \partial_{z}\left[\tilde{\chi}_{n}\left(\partial_{z}-F^{\prime}\right) \tilde{\chi}_{n}\right]+\tilde{\chi}_{n}^{2} .
\end{aligned}
$$

Then Eq. (6.6) can be rewritten as

$$
\langle\mathcal{H}\rangle_{\text {kink }}-\langle\mathcal{H}\rangle_{\text {vac }}-\partial_{z}\left[\mathcal{W}-\frac{1}{2} F^{\prime} \sum_{\omega_{n} \neq 0} \frac{\tilde{\chi}_{n}^{2}(z)}{2 \omega_{n}}\right]=\frac{1}{4} \partial_{z}^{2} \sum_{\omega_{n} \neq 0} \frac{\tilde{\chi}_{n}^{2}(z)}{2 \omega_{n}} .
$$

The soliton mass is given by the integral over the fiducial interval, i.e. over $z$ from $-L / 2+\Delta$ to $+L / 2-\Delta$ where $\Delta$ is chosen in such a way that $L \gg \Delta \gg m^{-1}$ (see the discussion in Sec. 3.9). Then it is not difficult to see that the right-hand side in Eq.(6.8) vanishes identically within the fiducial interval, and the soliton mass is given by the following formula

$$
M=\left[\mathcal{W}-\frac{1}{2} F^{\prime} \sum_{\omega_{n} \neq 0} \frac{\tilde{\chi}_{n}^{2}(z)}{2 \omega_{n}}\right]_{L / 2-\Delta}-\left[\mathcal{W}-\frac{1}{2} F^{\prime} \sum_{\omega_{n} \neq 0} \frac{\tilde{\chi}_{n}^{2}(z)}{2 \omega_{n}}\right]_{-L / 2+\Delta} .
$$

This result is perfectly analogous to what we had in the SSG model, except that the expression for the "central charge" changes, the one-loop correction to the soliton mass is given by

$$
\Delta M=\Delta\left\langle\mathcal{W}^{\prime \prime} \chi^{2}\right\rangle
$$

with the coefficient of $\left\langle\chi^{2}\right\rangle$ twice larger than it would be in a calculation of the correction to $\langle\mathcal{W}\rangle$ alone. (Note though that the classical part of $M$ is determined by $\Delta \mathcal{W}$.) As a result,

$$
M=2\left[m v^{2}-\frac{m}{4 \pi} \ln \frac{4 M_{r}^{2}}{m^{2}}\right] .
$$

This properly reproduces the part of the one-loop correction to the kink mass logarithmic in $M_{r}$ in the non-supersymmetric sine-Gordon model. The finite part is lost in the above reasoning because of our formal manipulations with divergent sums. This can be readily fixed by considering explicitly an ultraviolet regularization. The finite

\footnotetext{
${ }^{6}$ A similar behavior can be seen from Fig. 1: the integrals of the two curves over the whole box are
} equal, while the integrals over the fiducial interval are clearly different. 
contribution to the kink mass in fact is well known (see e.g. in [29]) and is equal to $-m /(2 \pi)$. We do not present details here, since the goal of this section is to illustrate the implementation of the supersymmetric (in the sense of Witten's quantum mechanics) boundary conditions and the separation of the kink energy from the energy of the boundaries.

\section{Anomaly and soliton mass in the SSG model at two loops}

We have shown in Sec. 3.6 that the anomaly relation (3.17) is not corrected beyond one loop. In the framework of the SSG model we now present two arguments confirming this statement.

The first is based on the relation for the superpotential in the SSG model

$$
\left\langle\mathcal{W}^{\prime \prime}(\phi)\right\rangle=-\frac{1}{v^{2}}\langle\mathcal{W}(\phi)\rangle
$$

which holds at all orders of perturbation theory. Using this relation in the expression for the anomaly, one finds, assuming that the one-loop expression for the anomaly is exact, that at

$$
v^{2}=\frac{1}{4 \pi}
$$

the central charge and also $\vartheta_{\mu}^{\mu}$ vanish and, thus, the theory becomes conformally invariant, which is the known behavior of the SSG model at this value of the coupling (see e.g. [32]).

The second confirmation comes from an explicit two-loop calculation of the kink mass, presented below, and its comparison with the known result [32 found under the assumption of factorizability of the $S$ matrix in this theory. The calculation of the kink mass in the SSG model at the multi-loop level was most recently considered in Ref. [14], where an actual two-loop calculation was also done in detail. We believe that our approach, based on the anomalous relation (3.17), provides us with a much simpler way of calculating the soliton mass, at least at the two-loop level. The calculation is presented in this section.

According to Eq. (3.17) the mass of the soliton in the SSG model is given by

$$
M=2\left\langle\mathcal{W}(\phi)+\frac{\mathcal{W}^{\prime \prime}(\phi)}{4 \pi}\right\rangle_{\pi v / 2}=2\left(1-\frac{1}{4 \pi v^{2}}\right)\langle\mathcal{W}(\phi)\rangle_{\pi v / 2}
$$

where the subscript $\pi v / 2$ denotes that the quantum average of the superpotential is taken over the "flat" vacuum at $\langle\phi\rangle=\pi v / 2$. The relation (7.1) is also taken into account in Eq. (7.2). Although the calculation of the quantum average of the superpotential function in two (or higher) loops is quite straightforward in any given regularization scheme in terms of the regulator parameter $M_{r}$ and the bare mass $m$, we prefer not 
to present it explicitly, since this part is completely absorbed in the renormalization of the particles' mass. Technically the reasoning is most straightforward for the on-shell fermion mass, which at the two loop level can be written as

$$
m_{F}=-\left\langle\mathcal{W}^{\prime \prime}(\phi)\right\rangle_{\pi v / 2}+\delta_{2} m=\frac{M}{2\left(v^{2}-\frac{1}{4 \pi}\right)}+\delta_{2} m
$$

where the equations (7.2) and (7.1) are used in the last equality, and $\delta_{2} m$ is the nontadpole contribution to the fermion self-energy given by a single graph of Fig. 2. The

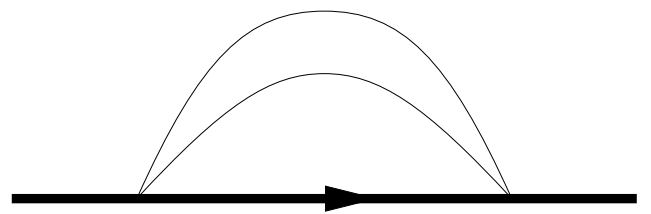

Figure 2: Non-tadpole two loop graph in the fermion self-energy. The heavy line denotes the fermion, and the thin lines denote bosons.

tadpole part of the mass, given by $\left\langle\mathcal{W}^{\prime \prime}(\phi)\right\rangle$ has no dispersion, while the finite graph of Fig. 2 introduces dispersion of the self-energy in fermion momentum $p$. For the on-shell mass of the fermion, we calculate this graph at $\not p=m$ and find:

$$
\delta_{2} m=-\left.\frac{m^{2}}{2 v^{4}} \int \frac{\mathrm{d}^{2} k}{(2 \pi)^{2}} \frac{\mathrm{d}^{2} q}{(2 \pi)^{2}} \frac{\not p+\not q+m}{(p+q)^{2}-m^{2}} \frac{1}{\left(k^{2}-m^{2}\right)} \frac{1}{(q+k)^{2}-m^{2}}\right|_{\not p=m}=-\frac{m}{96 v^{4}} .
$$

Thus, up to the two-loop terms, inclusively, (i.e. of the relative order $v^{-4}$ ) the mass of the kink is related to the renormalized on-shell mass $\bar{m}$ of the particles in the SSG model as

$$
M_{\text {two-loop }}=2 \bar{m}\left(v^{2}-\frac{1}{4 \pi}\right)+\frac{\bar{m}}{48 v^{2}} .
$$

This relation is in perfect agreement with the expansion, up to the appropriate order $\left(\gamma^{3}\right)$, of the known exact relation

$$
\bar{m}=2 M \sin \frac{\gamma}{16}
$$

with

$$
\frac{4}{\gamma}=v^{2}-\frac{1}{4 \pi}
$$

found [32] within the assumption of a factorized $S$ matrix in the SSG model.

\section{Extra superfields and extended supersymmetry}

In this section we will consider the $\mathcal{N}=2$ model in two dimensions, obtained by dimensional reduction of the four-dimensional Wess-Zumino model [19], and its deformations 
which break $\mathcal{N}=2$ down to $\mathcal{N}=1$. A general classification of $\mathcal{N}=2$ theories in two dimensions is given in 33.

In four dimensions the Wess-Zumino model realizes minimal, $\mathcal{N}=1$, supersymmetry with four fermionic generators. In two dimensions it means that an extra pair of fermionic generators is added to superalgebra (3.7). Let us briefly recall this construction putting emphasis on those elements that are specific for $\mathcal{N}=2$.

The Wess-Zumino model in four dimensions describes (self-)interactions of one complex scalar field $\varphi(x)$ and one complex Weyl spinor $\psi^{\alpha}(x), \alpha=1,2$. Both the boson and fermion degrees of freedom are united in one chiral superfield $\rceil$.

$$
\Phi\left(x_{L}, \theta\right)=\varphi\left(x_{L}\right)+\sqrt{2} \theta^{\alpha} \psi_{\alpha}\left(x_{L}\right)+\theta^{2} f\left(x_{L}\right),
$$

where $\theta$ is a two-component complex Grassmann spinor. The chiral coordinate $x_{L}$ is defined as

$$
\left(x_{L}\right)_{\alpha \dot{\alpha}}=x_{\alpha \dot{\alpha}}-2 i \theta_{\alpha} \bar{\theta}_{\dot{\alpha}}
$$

so that the supertransformation takes the form

$$
\theta_{\alpha} \rightarrow \theta_{\alpha}+\varepsilon_{\alpha}, \quad\left(x_{L}\right)_{\alpha \dot{\beta}} \rightarrow\left(x_{L}\right)_{\alpha \dot{\beta}}-4 i \theta_{\alpha} \bar{\varepsilon}_{\dot{\beta}},
$$

where $\alpha, \dot{\alpha}=1,2$ are the Lorentz indices. The Lagrangian of the Wess-Zumino model in terms of superfields is

$$
\mathcal{L}_{\mathrm{WZ}}=\frac{1}{4} \int d^{4} \theta \Phi \bar{\Phi}+\left\{\frac{1}{2} \int d^{2} \theta \mathcal{W}_{\mathrm{WZ}}(\Phi)+H . c .\right\}
$$

where $\mathcal{W}_{\mathrm{WZ}}$ is a superpotential. We choose it to be of the form

$$
\mathcal{W}_{\mathrm{WZ}}(\Phi)=\frac{i}{\sqrt{2}}\left[\frac{m^{2}}{4 \lambda} \Phi-\frac{2 \lambda}{3} \Phi^{3}\right]
$$

where $m$ and $\lambda$ are real parameters.

In components the Lagrangian takes the form

$$
\mathcal{L}_{\mathrm{WZ}}=\partial^{\mu} \varphi^{+} \partial_{\mu} \varphi+\psi^{\alpha} i \partial_{\alpha \dot{\alpha}} \bar{\psi}^{\dot{\alpha}}+f^{+} f+\left\{f \mathcal{W}_{\mathrm{WZ}}^{\prime}(\varphi)-\frac{1}{2} \mathcal{W}_{\mathrm{WZ}}^{\prime \prime}(\varphi) \psi^{\alpha} \psi_{\alpha}+\text { H.c. }\right\}
$$

where the auxiliary field $f$ can be eliminated by virtue of the classical equation of motion, $f^{+}=-\mathcal{W}_{\mathrm{WZ}}^{\prime}$.

Now, to perform the reduction to two dimensions it is sufficient to assume that all fields are independent of $x$ and $y$, they depend on $t$ and $z$ only. In two dimensions the Lagrangian can be presented in the following form:

$$
\mathcal{L}=\frac{1}{2}\left\{\partial_{\mu} \varphi_{i} \partial^{\mu} \varphi_{i}+i \bar{\psi}_{i} \gamma^{\mu} \partial_{\mu} \psi_{i}+f_{i} f_{i}+2 f_{i} \frac{\partial \mathcal{W}}{\partial \varphi_{i}}-\frac{\partial^{2} \mathcal{W}}{\partial \varphi_{i} \partial \varphi_{j}} \bar{\psi}_{i} \psi_{j}\right\} .
$$

\footnotetext{
${ }^{7}$ For our conventions and notation see Appendix A in Ref. [18]
} 
We introduced real fields $\varphi_{i}, \psi_{i}$ and $f_{i}$, where $i=1,2$. Up to normalization they are just real and imaginary parts of the original fields, e.g, $\varphi=\left(\varphi_{1}+i \varphi_{2}\right) / \sqrt{2}$. Summation over $i$ is implied. The superpotential $\mathcal{W}=2 \operatorname{Im} \mathcal{W}_{\mathrm{WZ}}$ is a function of two variables $\varphi_{1}$ and $\varphi_{2}$,

$$
\mathcal{W}\left(\varphi_{1}, \varphi_{2}\right)=\frac{m^{2}}{4 \lambda} \varphi_{1}-\frac{\lambda}{3} \varphi_{1}^{3}+\lambda \varphi_{1} \varphi_{2}^{2}
$$

The Lagrangian can also be presented in terms of $\mathcal{N}=1$ superfields $\Phi_{i}$ (see Eq. (2.3) for the definition), namely

$$
\mathcal{L}=i \int \mathrm{d}^{2} \theta\left\{\frac{1}{4} \bar{D}_{\alpha} \Phi_{i} D_{\alpha} \Phi_{i}+\mathcal{W}\left(\Phi_{1}, \Phi_{2}\right)\right\}
$$

The presence of extended supersymmetry is reflected in the harmonicity of this superpotential,

$$
\frac{\partial^{2} \mathcal{W}}{\partial \varphi_{i} \partial \varphi_{i}}=0 \quad \text { for } \mathcal{N}=2
$$

We will consider a more general case of nonharmonic $\mathcal{W}\left(\varphi_{1}, \varphi_{2}\right)$,

$$
\mathcal{W}\left(\varphi_{1}, \varphi_{2}\right)=\frac{m^{2}}{4 \lambda} \varphi_{1}-\frac{\lambda}{3} \varphi_{1}^{3}+q \lambda \varphi_{1} \phi_{2}^{2}+\frac{p m}{2} \varphi_{2}^{2},
$$

where $p$ and $q$ are dimensionless parameters. For $q \neq 1, p \neq 0$ the extended $\mathcal{N}=2$ supersymmetry is explicitly broken down to $\mathcal{N}=1$.

The classical solution for the kink is the same as in the SPM model with one superfield considered earlier; the second field $\varphi_{2}$ vanishes,

$$
\varphi_{1}=\phi_{0}(z)=\frac{m}{2 \lambda} \tanh \frac{m z}{2}, \quad \varphi_{2}=0 .
$$

The topological current $\zeta^{\mu}$ now takes the form,

$$
\zeta^{\mu}=\epsilon^{\mu \nu} \partial_{\nu}\left[\mathcal{W}+\frac{1}{4 \pi} \frac{\partial^{2} \mathcal{W}}{\partial \varphi_{i} \partial \varphi_{i}}\right] .
$$

For the harmonic superpotentials, i.e. $\mathcal{N}=2$, the anomaly vanishes.

The quadratic expansion of the energy density $\mathcal{H}$ around the solution (8.12) looks as follows (compare with Eq. (3.45)):

$$
\begin{aligned}
& {\left[\mathcal{H}-\zeta^{0}\right]_{\text {quad }}=} \\
& \frac{1}{2}\left\{\dot{\chi}_{1}^{2}+\left[\left(\partial_{z}+2 \lambda \phi_{0}\right) \chi_{1}\right]^{2}+i \eta_{1}\left(\partial_{z}-2 \lambda \phi_{0}\right) \xi_{1}+i \xi_{1}\left(\partial_{z}+2 \lambda \phi_{0}\right) \eta_{1}\right. \\
& \quad+\dot{\chi}_{2}^{2}+\left[\left(\partial_{z}-2 q \lambda \phi_{0}-p m\right) \chi_{2}\right]^{2}+i \eta_{2}\left(\partial_{z}+2 q \lambda \phi_{0}+p m\right) \xi_{2} \\
& \left.\quad+i \xi_{2}\left(\partial_{z}-2 q \lambda \phi_{0}-p m\right) \eta_{2}\right\}
\end{aligned}
$$

where the following notation is used:

$$
\chi_{1}=\varphi_{1}-\phi_{0}, \quad \chi_{2}=\varphi_{2}, \quad\left(\begin{array}{c}
\xi_{i} \\
\eta_{i}
\end{array}\right)=\psi_{i}
$$


The part of this expression containing the fields marked by the subscript 1 is exactly the same as in Eq. (3.45); the fields with the subscript 2 are new.

What changes do these new fields introduce? First of all, there is a new fermionic zero mode in the field $\xi_{2}$,

$$
\xi_{2}^{\text {zero }}=\frac{\exp (-p m z)}{\cosh ^{2 q}(m z / 2)} .
$$

At $p=0, q=1$ it has the same functional form as the old fermionic mode in $\eta_{1}$. This is not surprising because $\mathcal{N}=2$ supersymmetry places the kink in the supermultiplet which contains two bosonic and two fermionic solitons (the second bosonic kink corresponds to both fermionic modes filled in). What is more intriguing is that the same degeneracy remains after $\mathcal{N}=2$ breaking. If $p \neq 0$ and/or $q \neq 1$ the $\mathcal{N}=2$ SUSY is replaced by $\mathcal{N}=1$. Still, two fermion zero modes are present. The Jackiw-Rebbi index [34] provides a general argument proving the existence of the zero mode in $\xi_{2}$. Thus, we have two degenerate fermionic solitons, and two bosonic. The degeneracy is true only for the ground solitonic states. The $\mathcal{N}=2$ degeneracy of the nonzero modes is lifted at $p \neq 0$ and/or $q \neq 1$.

For the nonzero modes of $\chi_{2}, \xi_{2}$ and $\eta_{2}$ two new differential operators come into play,

$$
\begin{aligned}
& -\left(\partial_{z}-2 q \lambda \phi_{0}-p m\right)\left(\partial_{z}+2 q \lambda \phi_{0}+p m\right) v_{n}(z)=\tilde{\omega}_{n}^{2} v_{n}(z), \\
& -\left(\partial_{z}+2 q \lambda \phi_{0}+p m\right)\left(\partial_{z}-2 q \lambda \phi_{0}-p m\right) \tilde{v}_{n}(z)=\tilde{\omega}_{n}^{2} \tilde{v}_{n}(z),
\end{aligned}
$$

Again, at $p=0, q=1$ they coincide with the operators of Eqs. (3.46) and (3.49), due to $\mathcal{N}=2$. This degeneracy is lifted in the general case, but the degeneracy of the eigenvalues of the operators (8.17) stays intact. Their eigenmodes are related,

$$
\tilde{v}_{n}(z)=\frac{1}{\tilde{\omega}_{n}}\left(\partial_{z}+2 q \lambda \phi_{0}+p m\right) v_{n}(z)
$$

Expanding $\xi_{2}$ in $v_{n}(z), \chi_{2}, \eta_{2}$ in $\tilde{v}_{n}(z)$ and quantizing the coefficients in the same way as in Sec. 3.7 we observe the explicit fermion-boson cancellation in the expectation value over the soliton state,

$$
\left\langle\operatorname{sol}\left|\mathcal{H}(x)-\zeta^{0}(x)\right| \operatorname{sol}\right\rangle=0 .
$$

The general consideration in Sec. 3.7 proves this to all orders. It is important that in proving the fermion-boson cancellation no integration by parts is made. Thus, the statement of cancellation is insensitive to the particular choice of the boundary conditions. (As in Sec. 3.7, they must be $\mathcal{N}=1$ supersymmetric, of course.)

So far everything looks very similar to the minimal model treated in Secs. 2 and 3. The only distinction is the complex structure inherent to the theory with four supercharges. This structure is essential, it is just this complexification that leads to the nonrenormalization theorem for the superpotential and for the soliton mass. 
The relation (8.19) makes finding the one-loop quantum correction a simple job, similar to the one performed in Sec. 1. A somewhat novel feature is that the quantum fluctuations of $\chi_{2}$ at $z \rightarrow \infty$ and $z \rightarrow-\infty$ are different,

$$
\left\langle\chi_{2}^{2}(z \rightarrow \pm \infty)\right\rangle=\frac{1}{4 \pi} \ln \frac{4 M_{r}^{2}}{m^{2}(q \pm p)^{2}} .
$$

The result for the soliton mass at one loop is

$$
M=\frac{1}{6} \frac{m^{3}}{\lambda^{2}}-(1-q) \frac{m}{4 \pi}\left[\ln \frac{4 M_{r}^{2}}{m^{2}}+2\right]-\frac{m}{8 \pi}\left[q \ln \left(q^{2}-p^{2}\right)^{2}+p \ln \frac{(q+p)^{2}}{(q-p)^{2}}\right],
$$

The generalization to the case of arbitrary $\mathcal{W}\left(\varphi_{1}, \varphi_{2}\right)$ presents no problem,

$$
M=\left\{\mathcal{W}+\sum_{k=1,2} \frac{\mathcal{W}_{k k}}{8 \pi}\left[\ln \frac{4 M_{r}^{2}}{\mathcal{W}_{k k}^{2}}+2\right]\right\}_{\{\varphi\}=\left\{\phi_{0}(z=+\infty)\right\}}-\left\{\{\varphi\}=\left\{\phi_{0}(z=-\infty)\right\}\right\}
$$

where

$$
\mathcal{W}_{k l}=\frac{\partial^{2} \mathcal{W}}{\partial \varphi_{k} \partial \varphi_{l}}
$$

and $\left\{\phi_{0}(z)\right\}$ denotes the kink solution, $\varphi_{1}(z)=\phi_{0}(z), \varphi_{2}(z)=0$. We see that there is no correction in the case of unbroken $\mathcal{N}=2$ supersymmetry, $\sum \mathcal{W}_{k k}=0$, as it should be due to nonrenormalization of the superpotential.

In the case of the soft breaking by the mass term,

$$
\mathcal{W}=\mathcal{W}_{\text {harmonic }}+\frac{1}{2} \mu \phi_{2}^{2}, \quad \mathcal{W}_{22}=-\mathcal{W}_{11}+\mu
$$

there is no ultraviolet divergence in the correction to the soliton mass. Moreover, the anomaly, presented by the term 2 in the square brackets, also goes away. Indeed, these contributions are proportional to $\mathcal{W}_{11}+\mathcal{W}_{22}=\mu$ and are the same at both boundaries $z= \pm \infty$.

It means that we can treat the field $\Phi_{2}$ as the ultraviolet regulator for $\Phi_{1}$, provided the $\Phi_{2}$ mass is much larger than that of $\Phi_{1}$, i.e. $\mu \gg m_{1}$. In this limit we get

$$
M=\left\{\mathcal{W}+\frac{\mathcal{W}_{11}}{8 \pi}\left[\ln \frac{\mu^{2}}{\mathcal{W}_{11}^{2}}+2\right]\right\}_{\varphi_{1}=\phi_{0}(z=+\infty)}-\left\{\varphi_{1}=\phi_{0}(z=-\infty)\right\} .
$$

In this way we reproduce again the result (4.5) for the soliton mass including the anomalous part (up to the substitution $2 M_{r} \rightarrow \mu$ ).

\section{Discussion}

The residual supersymmetry in the soliton background field entails a mode degeneracy [2], in much the same way as in the four-dimensional problem of instantons. This 
degeneracy manifests itself in the complete cancellation of the quantum corrections, order by order in the (small) coupling constant. In the soliton problem this cancellation takes place in the quantity $\langle\mathrm{sol}|H-\mathcal{Z}|$ sol $\rangle$. The soliton mass coincides with the central charge to all orders. This property, once established at the classical level, cannot disappear at the level of quantum corrections, at least in the weak coupling regime.

Surprisingly, this question has caused an ongoing debate in the literature. In many works, including some recent ones, it is asserted that BPS saturation is lost at the one-loop level. Our conclusions are categorically against this point of view. The reason explaining the discrepancy between the (independently calculated in the literature) soliton mass and central charge is the omission of the anomalous term in $\mathcal{Z}$. We have established the existence of the anomaly (3.16) by four independent methods. Although our explicit calculations were carried out in one loop, we argue that Eq. (3.16) is valid in the operator form to all orders. In both models considered, SPM and SSG, the contribution of the anomaly in the central charge is negative, it is finite (i.e. contains no ultraviolet logarithms) and makes $\mathcal{Z}$ smaller. Without the anomalous contribution, the would-be value of $\mathcal{Z}$ apparently exceeds $M_{\text {sol }}$ (e.g. [14]), in sharp contradiction with the general principles. In fact, Ref. [14] carries a special section intended to reconcile the apparent contradiction between the general requirement that $M_{\text {sol }} \geq \mathcal{Z}$ and the concrete result of [14] according to which $M_{\text {sol }}<\mathcal{Z}$. Needless to say that the contradiction is automatically eliminated once the anomaly is taken into account.

To the best of our knowledge, the anomaly in the central charge in two-dimensional models with minimal supersymmetry was not discussed previously. However, indirectly its existence could have been inferred from well-known results. For instance, the inspection of the exact $S$ matrix in the SSG model (e.g. [32]) shows that the parameter $v^{2}$ in the superpotential (see Eq. (2.9)) and an analogous parameter in the on-shell scattering amplitudes are not identical, the latter is different from the former by a finite renormalization. In our language this finite renormalization can be traced back to the anomaly.

Much of the controversy in the literature is focussed around the boundary conditions. It is not uncommon to choose them in a non-supersymmetric manner, e.g. [3, 13, 14]. It is quite obvious that Eq. (17) in [14] is incompatible with the residual supersymmetry, see Eq. (2.20). Then the manifest cancellation of the boson and fermion corrections is lost, and one has to calculate separately the boson and fermion correction. We hasten to add that such an approach a priori is fully legitimate, there is nothing wrong with it. Moreover, the value of the one-loop soliton mass obtained in this way in Refs. [14, 15] and some previous works is perfectly correct. The non-SUSY approach to the kink mass calculation has its advantages since the calculation is based on the dynamics in the bulk, and the problem of untangling the "boundary condition contamination" essentially does not arise. On the other hand, it totally misses all advantages provided by supersymmetry.

The approach we follow, which exploits in full the advantages of supersymmetry, is very economic. Nothing comes for free, however. In the SUSY-based approach there is a subtle point. If one considers just the soliton mass per se, i.e. the total energy 
of the system in the box, the boundary conditions may have a drastic impact on the result. They distort the soliton state under consideration in the domain adjacent to the boundary, see Sec. 3.9. For instance, if the boundary conditions on $\chi$ at $z= \pm L / 2$ are periodic, they force $\langle\chi( \pm L / 2)\rangle$ to be the same while in the undistorted soliton $\langle\chi(L / 2)\rangle$ and $\langle\chi(-L / 2)\rangle$ have different signs. This distortion happens even with the supersymmetric boundary conditions. For instance, in [12] the following condition on the derivative of $\chi$ was suggested: $\left(\partial_{z}+F^{\prime}\right) \chi(L / 2)=-\left(\partial_{z}+F^{\prime}\right) \chi(-L / 2)$, along with the antiperiodic boundary condition on $\chi$, compatible with $(2.20)$. In this case the boundary conditions enforce $\langle\bar{\psi} \psi\rangle_{L / 2}=\langle\bar{\psi} \psi\rangle_{-L / 2}$, while for the undistorted soliton $\langle\bar{\psi} \psi\rangle_{L / 2}$ must be equal to $-\langle\bar{\psi} \psi\rangle_{-L / 2}$.

It is easy to see that the contamination of the soliton near the boundaries adds to its mass a quantity of the same order of magnitude as the one-loop correction. Therefore, it is not so surprising that a spectrum of predictions for the corrected soliton mass is obtained. These predictions refer to the contaminated soliton, rather than the genuine ground-state soliton. It is even conceivable to design a state whose mass at one loop is exactly equal to the classical soliton mass. To this end one requires $\chi$ and $\psi_{2}$ to vanish at the boundary. This is a possible boundary condition, consistent with (2.20). This sensitivity to the boundary conditions is due to the fact that the soliton mass is expressed, via SUSY, in terms of the central charge, a quantity defined at the boundary.

It is clear that the problem with the near-boundary domain is totally unphysical. The effects due to the finite-size box must be cut off and discarded. The physical ground-state soliton is certainly insensitive to the conditions we impose infinitely far away from its center. To solve this problem it is advantageous to consider the local form of BPS saturation condition, see Eq. (3.42). Then, by inspecting the (one-loop corrected) soliton profile, or the energy distribution, we can easily figure out which contribution to the mass comes from the undistorted soliton and which is related to distortions near the boundary. The latter must not be included. This approach is close to that suggested in [10] long ago, the work which was in essence ignored in the later publications. Conceptually, we do the same; certainly, we do include the anomaly omitted in Ref. [10]. We expand this approach in various directions, e.g. we consider the soliton profile in the minimal (one-superfield) model, and discuss a two-superfield model presenting a deformation of the extended $\mathcal{N}=2$ model.

If one is not interested in the soliton profile, one can calculate the soliton mass per se from the central charge, by shifting the calculation of the latter a little bit, from the boundary of the box, inside the fiducial domain. The edge of the fiducial domain is still (infinitely) far from the soliton center, and, hence, the central charge is determined by the flat vacuum calculation.

In the $\mathcal{N}=2$ models the classical expression for the soliton mass stays intact there are no quantum corrections. The corresponding nonrenormalization theorem is in essence identical to that valid for the four-dimensional domain walls 17,18 . The $\mathcal{N}=2$ SUSY can be broken softly down to $\mathcal{N}=1$ by a mass term of one of the superfields. The one-loop correction to the central charge then is finite, since the mass term plays the role of the ultraviolet regulator. The expression for the soliton mass is in full accord 
with the general relation (3.16).

One last remark concerning the recent publications $[14,15]$. To an extent, our study was inspired by the thorough analysis of Ref. [14], which clearly exhibited the problem: the kink mass received a correction while the central charge stayed intact. In our view, the calculations presented in [14] are correct. Our explanation of the results obtained in [14] is that they refer to the Hamiltonian corrected by the anomaly and to the uncorrected central charge. These quantities, however, are not the ones related by supersymmetry. The authors of the recent paper [15] claim a double success: the correct value of the kink mass as well as the BPS saturation. Their calculation of the kink mass is quite instructive. In particular, it indirectly hints at the anomaly in the form of the relation $k \tan \left(\delta_{B}-\delta_{F}\right)=m$ between the boson $\left(\delta_{B}\right)$ and fermion $\left(\delta_{F}\right)$ phase shifts. As for the central charge, it was fixed in [15] to be equal to the kink mass by supersymmetry, the assertion ascending to Ref. [4]. The topological nature of the

charge was not considered. In this way the authors avoided addressing the problem of the difference between the uncorrected and corrected forms of the central charge. It was implied that renormalization fixes the central charge uniquely - this is the point we cannot accept.

The BPS saturation, as we have demonstrated, is a direct consequence of the surviving supersymmetry. It cannot be broken as long as this symmetry is maintained. The presence of the anomalous term in the central charge fully resolves the long-standing riddle.

\section{Acknowledgments}

M.S. and A.V. would like to thank I. Kogan for a valuable contribution at an initial stage of this work. Discussions with B. Chibisov, R.L. Jaffe, A. Larkin, A. Marshakov and A. Ritz are gratefully acknowledged.

This work was supported in part by DOE under the grant number DE-FG0294ER40823. 


\section{References}

[1] E. Witten and D. Olive, Phys. Lett. B78 (1978) 97.

[2] A. D'Adda and P. Di Vecchia, Phys. Lett. B73 (1978) 162.

[3] R.K. Kaul and R. Rajaraman, Phys. Lett. B131 (1983) 357.

[4] C. Imbimbo and S. Mukhi, Nucl. Phys. B247 (1984) 471.

[5] A. D'Adda, R. Horsley, and P. Di Vecchia, Phys. Lett. B76 (1978) 298; R. Horsley, Nucl. Phys. B151 (1979) 399.

[6] J.F. Schonfeld, Nucl. Phys. B161 (1979) 125.

[7] S. Rouhani, Nucl. Phys. B182 (1981) 462.

[8] A. Uchiyama, Nucl. Phys. B244 (1984) 57; Prog. Theoret. Phys. 75 (1986) 1214.

[9] A. Uchiyama, Nucl. Phys. B278 (1986) 121.

[10] H. Yamagishi, Phys. Lett. B147 (1984) 425.

[11] A.K. Chatterjee and P. Majumdar, Phys. Lett. B159 (1985) 37.

[12] A.K. Chatterjee and P. Majumdar, Phys. Rev. D30 (1984) 844.

[13] A. Rebhan and P. Nieuwenhuizen, Nucl. Phys. B508 (1997) 449.

[14] H. Nastase, M. Stephanov, P. Nieuwenhuizen, and A. Rebhan, Topological boundary conditions, the BPS bound, and elimination of ambiguities in the quantum mass of solitons, hep-th/9802074; Nucl. Phys. B, to appear.

[15] N. Graham and R.L. Jaffe, Energy, Central Charge, and the BPS Bound for 1+1 Dimensional Supersymmetric Solitons, hep-th/9808140.

[16] V. Novikov, M. Shifman, A. Vainshtein and V. Zakharov, Nucl. Phys. B229 (1983) 381; Phys. Lett. B166 (1986) 329.

[17] G. Dvali and M. Shifman, Nucl. Phys. B504 (1997) 127.

[18] B. Chibisov and M. Shifman, Phys. Rev. D56 (1997) 7990.

[19] J. Wess and B. Zumino, Phys. Lett. B49 (1974) 52.

[20] J. Iliopoulos and B. Zumino, Nucl. Phys. B76 (1974) 310; P. West, Nucl. Phys. B106 (1976) 219; M. Grisaru, W. Siegel, and M. Roček, Nucl. Phys. B159 (1979) 429 .

[21] M.B. Voloshin, Phys. Rev. D47 (1993) 357. 
[22] B.H. Smith, Phys. Rev. D47 (1993) 3518.

[23] M. Shifman, Phys. Reports 209 (1991) 341.

[24] V. Novikov et al., Nucl. Phys. B229 (1983) 394.

[25] E. Witten, Nucl. Phys. B202 (1982) 253.

[26] M. Shifman and A. Vainshtein, Nucl. Phys. B277 (1986) 456.

[27] L. Gendenshtein and I. Krive, Usp. Fiz. Nauk 146 (1985) 553 [Sov. Phys. Uspekhi 28 (1985) 645].

[28] F. Schwabl, Quantum Mechanics, Springer-Verlag, Berlin, 1988.

[29] R. Rajaraman, Solitons and Instantons, North-Holland, Amsterdam, 1982.

[30] M.B. Voloshin, Phys. Rev. D47 (1993) 2573.

[31] L.S. Brown, Phys. Rev. D46 (1992) 4125.

[32] R. Shankar and E. Witten, Phys. Rev. D17 (1978) 2134;

A.M. Tsvelik, Yad. Fiz. 47 (1988) 272 [Sov. J. Nucl. Phys. 47 (1988) 172];

P. Fendley and K. Intriligator, Nucl. Phys. B372 (1990) 533;

C. Ahn, D. Bernard and A. LeClair, Nucl. Phys. B346 (1990) 409;

C. Ahn, Nucl. Phys. B354 (1991) 57.

[33] S. Cecotti and C. Vafa, Commun. Math. Phys. 158 (1993) 569.

[34] R. Jackiw and C. Rebbi, Phys. Rev. D13 (1976) 3398. 